Fluvial Egg Drift Simulator (FluEgg) User's Manual

Open-File Report 2021-1052 



\section{Fluvial Egg Drift Simulator (FluEgg) User's Manual}

By Marian M. Domanski, Jessica Z. LeRoy, Michael Berutti, and P. Ryan Jackson

Open-File Report 2021-1052 


\section{U.S. Geological Survey, Reston, Virginia: 2021}

For more information on the USGS - the Federal source for science about the Earth, its natural and living resources, natural hazards, and the environment—visit https://www.usgs.gov or call 1-888-ASK-USGS.

For an overview of USGS information products, including maps, imagery, and publications, visit https://store.usgs.gov/.

Any use of trade, firm, or product names is for descriptive purposes only and does not imply endorsement by the U.S. Government.

Although this information product, for the most part, is in the public domain, it also may contain copyrighted materials as noted in the text. Permission to reproduce copyrighted items must be secured from the copyright owner.

Suggested citation:

Domanski, M.M., LeRoy, J.Z., Berutti, M., and Jackson, P.R., 2021, Fluvial Egg Drift Simulator (FluEgg) user's manual: U.S. Geological Survey Open-File Report 2021-1052, 30 p., https://doi.org/10.3133/ofr20211052.

Associated Data:

Domanski, M.M., and Berutti, M.C., 2020, FluEgg: U.S. Geological Survey software release, https://doi.org/10.5066/ P93UCQR2.

ISSN 2331-1258 (online) 


\section{Acknowledgments}

The work was funded by the Great Lakes Restoration Initiative as administered by the U.S. Environmental Protection Agency. We thank Tatiana Garcia, Elizabeth Murphy, and Lori Jones for assistance with early drafts of this manual. 



\section{Contents}

Acknowledgments ……...................................................................................................................

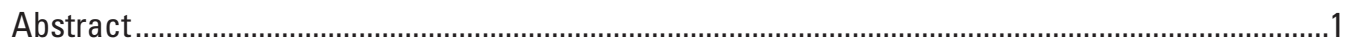

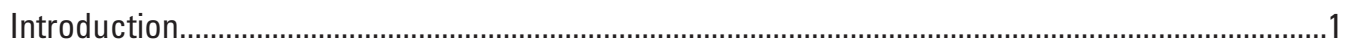

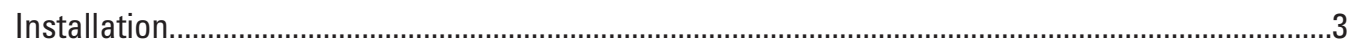

Graphical User Interface for the Fluvial Egg Drift Simulator (FluEgg) ................................................

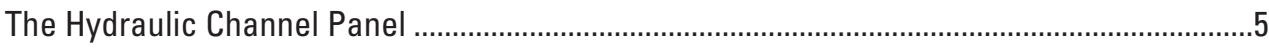

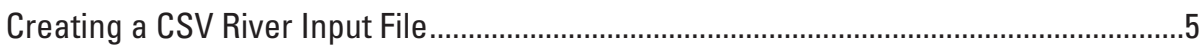

Preparing a HEC-RAS Project for Use in FluEgg ……......................................................

Loading the Hydraulic Channel .......................................................................................

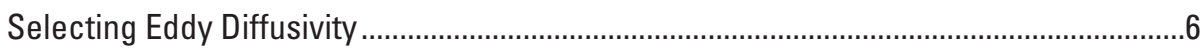

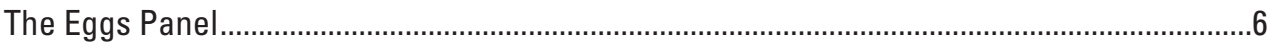

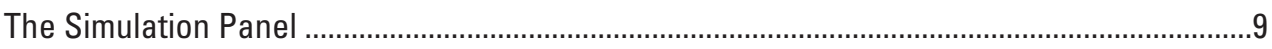

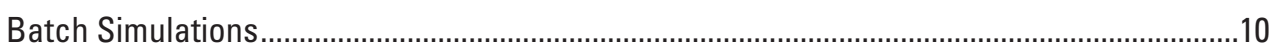

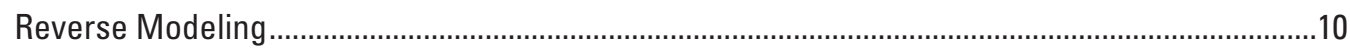

Plotting and Post-Processing Results ....................................................................................

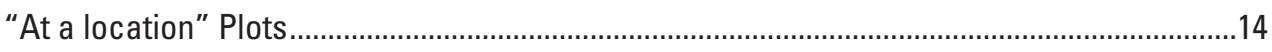

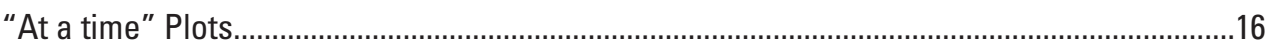

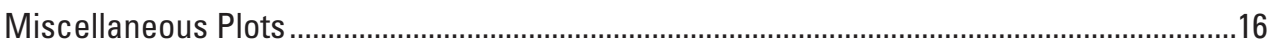

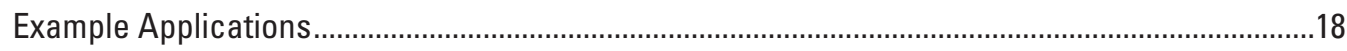

Running FluEgg with a CSV River Input File .................................................................

Running FluEgg with a Steady HEC-RAS Project ...........................................................19

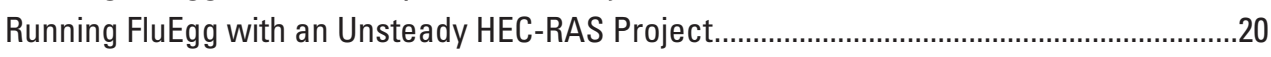

Generating KML Outputs in FluEgg — Illinois River Example ...............................................21

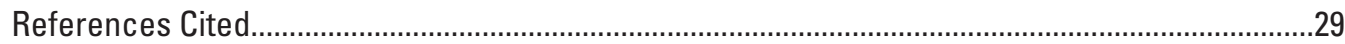

\section{Figures}

1. Conceptual schematic of the hydraulic and biologic processes modeled in the Fluvial Egg Drift Simulator ...........................................................................................2

2. Screenshot of the Fluvial Egg Drift Simulator home window before loading

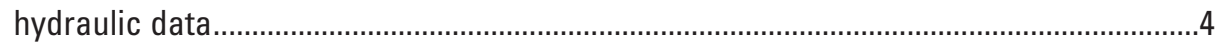

3. Screenshot of a simple example of a river input file with five cells ................................6

4. Screenshot of the Hydrologic Engineering Center's River Analysis System settings subinterface with the Fluvial Egg Drift Simulator...............................................6

5. Screenshot of the Fluvial Egg Drift Simulator home window after loading river input parameters..........................................................................................................7

6. Image showing the Fluvial Egg Drift Simulator coordinate system when $\mathrm{Y}$ and $\mathrm{Z}$ are fractional distances or entered in meters ................................................................

7. Screenshot of the "Egg" characteristics panel within the Fluvial Egg Drift Simulator if the user selects the "Constant $\rho$, $d$ " option. 2 6 6

\section{7} 8

8. Screenshot of the warning message generated by the Fluvial Egg Drift Simulator informing the user that a smaller time step must be used in the simulation. 
9. Screenshot of The Fluvial Egg Drift Simulator home window after a successful simulation

10. Screenshot of the batch simulation window within the Fluvial Egg Drift Simulator .......12

11. Screenshot of an example batch simulation setup within the Fluvial Egg Drift Simulator

12. Screenshot of an example of the batch simulation output within the Fluvial Egg Drift Simulator.

13. Graphs showing two useful plots for identifying potential spawning locations using output from the Fluvial Egg Drift Simulator.

14. Screenshots showing the "Plots" window within the Fluvial Egg Drift Simulator ..........15

15. Graph showing example of the "Combined Eggs Past Location v. Time" plot within the Fluvial Egg Drift Simulator

16. Graphs showing example of the "Distribution of Eggs" plots within the Fluvial Egg Drift Simulator.

17. Graph showing example of the "Location Quantiles Over Time" plot for the $\mathrm{X}$-axis within the Fluvial Egg Drift Simulator.

18. Graph showing distribution of eggs in the streamwise direction at 30 hours within the Fluvial Egg Drift Simulator

19. Screenshot of Hydrologic Engineering Center's River Analysis System Settings window for the steady flume example within the Fluvial Egg Drift Simulator.....

20. Screenshot of egg and simulation parameters for the steady flume example within the Fluvial Egg Drift Simulator

21. Graph showing the resulting distribution of grass carp eggs at the hatching time in the steady flume example with a water temperature of 20 degrees Celsius within the Fluvial Egg Drift Simulator

22. Graph showing the simple hydrograph used for the unsteady flume example within the Fluvial Egg Drift Simulator

23. Screenshot of Hydrologic Engineering Center's River Analysis System Settings for the unsteady flume example within the Fluvial Egg Drift Simulator.

24. Screenshot of egg and simulation parameters for the unsteady flume example within the Fluvial Egg Drift Simulator

25. Graph showing the resulting longitudinal distribution of grass carp eggs at the hatching time in the unsteady flume example with a water temperature of 22 degrees Celsius within the Fluvial Egg Drift Simulator.

26. Screenshot of FluEgg home window with egg and simulation parameters for Illinois River example within the Fluvial Egg Drift Simulator

27. Screenshot of plotting window for exporting a Keyhole Markup Language file of the plume quantiles at 30 hours after spawning for the Illinois River example within the Fluvial Egg Drift Simulator

\section{Table}

1. Data for example of comma-separated values river input file 


\section{Conversion Factors}

International System of Units to U.S. customary units

\begin{tabular}{lcl}
\hline \multicolumn{1}{c}{ Multiply } & By & \multicolumn{1}{c}{ To obtain } \\
\hline meter $(\mathrm{m})$ & Length & \\
kilometer $(\mathrm{km})$ & 3.281 & foot $(\mathrm{ft})$ \\
meter $(\mathrm{m})$ & 0.6214 & mile $(\mathrm{mi})$ \\
\hline & 1.094 & yard $(\mathrm{yd})$ \\
\hline meter per second $(\mathrm{m} / \mathrm{s})$ & Flow rate & \\
cubic meter per second $\left(\mathrm{m}^{3} / \mathrm{s}\right)$ & 3.281 & foot per second $(\mathrm{ft} / \mathrm{s})$ \\
\hline & 35.31 & cubic foot per second $\left(\mathrm{ft}^{3} / \mathrm{s}\right)$ \\
\hline kilogram per cubic meter $\left(\mathrm{kg} / \mathrm{m}^{3}\right)$ & Density & \\
gram per cubic centimeter $\left(\mathrm{g} / \mathrm{cm}^{3}\right)$ & 0.06242 & pound per cubic foot $\left(\mathrm{lb} / \mathrm{ft}^{3}\right)$ \\
\hline
\end{tabular}

Temperature in degrees Celsius $\left({ }^{\circ} \mathrm{C}\right)$ may be converted to degrees Fahrenheit $\left({ }^{\circ} \mathrm{F}\right)$ as ${ }^{\circ} \mathrm{F}=(1.8 \times$ $\left.{ }^{\circ} \mathrm{C}\right)+32$.

Temperature in degrees Fahrenheit $\left({ }^{\circ} \mathrm{F}\right)$ may be converted to degrees Celsius $\left({ }^{\circ} \mathrm{C}\right)$ as ${ }^{\circ} \mathrm{C}=\left({ }^{\circ} \mathrm{F}-\right.$ 32) / 1.8 .

\section{Abbreviations}

1D one-dimensional

3D three-dimensional

CSV comma-separated values

FluEgg Fluvial Egg Drift Simulator

$\mathrm{GBI} \quad$ gas bladder inflation

GUI graphical user interface

HEC-RAS Hydrologic Engineering Center's River Analysis System

KML Keyhole Markup Language

USGS U.S. Geological Survey 



\title{
Fluvial Egg Drift Simulator (FluEgg) User's Manual
}

\author{
By Marian M. Domanski, ${ }^{1}$ Jessica Z. LeRoy, ${ }^{1}$ Michael Berutti, ${ }^{2}$ and P. Ryan Jackson ${ }^{1}$
}

\section{Abstract}

The Fluvial Egg Drift Simulator (FluEgg) was developed to simulate the transport and dispersion of invasive carp eggs and larvae in a river. FluEgg currently (2020) supports modeling of bighead carp (Hypophthalmichthys nobilis), silver carp (H. molitrix), and grass carp (Ctenopharyngodon idella), with the planned addition of black carp (Mylopharyngodon piceus) once developmental data are available. FluEgg integrates the biological development of invasive carp eggs and larvae with a particle transport model that simulates the advection and dispersion of the eggs and larvae based on user-supplied one-dimensional hydraulic conditions. FluEgg can be used to evaluate the hydrodynamic suitability of a river for invasive carp spawning, to inform sampling and monitoring efforts, and to identify the most likely spawning areas of captured eggs or larvae.

\section{Introduction}

The Fluvial Egg Drift Simulator (FluEgg) is a biophysical Lagrangian particle transport model that simulates the transport and dispersion of individual fertilized invasive carp eggs and larvae in a river (Garcia and others, 2013, 2015; Murphy and others, 2016). FluEgg currently (2020) supports modeling of bighead carp (Hypophthalmichthys nobilis), silver carp (H. molitrix), and grass carp (Ctenopharyngodon idella), with the planned addition of black carp (Mylopharyngodon piceus) once developmental data are available. FluEgg was designed to help answer questions regarding management and control strategies for invasive carp in early life stages; specifically, FluEgg was intended to evaluate if the hydrodynamics of a subject river are sufficient to maintain negatively buoyant invasive carp eggs in suspension in the water column until hatching given limited data and no hydraulic model of the subject river. FluEgg can be used to evaluate the hydrodynamic suitability of a river for invasive carp spawning, to inform sampling and monitoring efforts, and to identify the

\footnotetext{
${ }^{1}$ U.S. Geological Survey.

${ }^{2}$ Self-employed, Urbana, Illinois, under contract to the U.S. Geological Survey.
}

most likely spawning areas of captured eggs or larvae. The FluEgg model is written in the Python ${ }^{\circledR}$ programming language (Python Software Foundation, Wilmington, Delaware, United States).

FluEgg integrates the biological development of invasive carp eggs and larvae with a particle transport model that simulates the advection and dispersion of the eggs and larvae based on user-supplied one-dimensional (1D) hydraulic conditions (Garcia and others, 2013, 2015; Murphy and others, 2016). A river channel in FluEgg is represented as a series of discrete cells with rectangular cross-sections and constant hydraulic parameters. The user must provide as input the length, depth, discharge, average velocity, shear velocity, and water temperature for each cell. FluEgg combines these 1D cell-averaged data with open channel flow theory to build a three-dimensional (3D) model domain with no plan-form geometry (meaning the channel is straight) in which the cells are aligned along their centerline (fig. 1).

To determine the local velocity at the position of an egg or larva within the 3D model domain, the average velocity for each cell is used to estimate the lateral (transverse/crossstream) and vertical profiles of longitudinal (streamwise) velocity in each cell using open channel flow theory and empirical relations (Garcia and others, 2013, 2015). The variation in streamwise velocity over the vertical water column is constructed using a logarithmic velocity profile of the following form:

$$
\frac{u(z)}{u_{*}}=\frac{1}{\kappa} \ln \left(\frac{z}{h_{0}}\right)+8.5
$$

where

$$
\begin{aligned}
& u(z) \quad \text { is the longitudinal velocity, in meters } \\
& \text { per second, at a height } z \text {, in meters, } \\
& \text { above the bed; } \\
& u_{*} \quad \text { is the shear velocity, in meters per second; } \\
& \kappa \quad \text { is the von Kármán constant }(0.41), \\
& \text { unitless; and } \\
& h_{0} \quad \text { is the roughness height, in meters. }
\end{aligned}
$$

The roughness height is determined using the following equation:

$$
h_{0}=\frac{11 h}{\exp \left(\kappa u_{*} U\right)}
$$




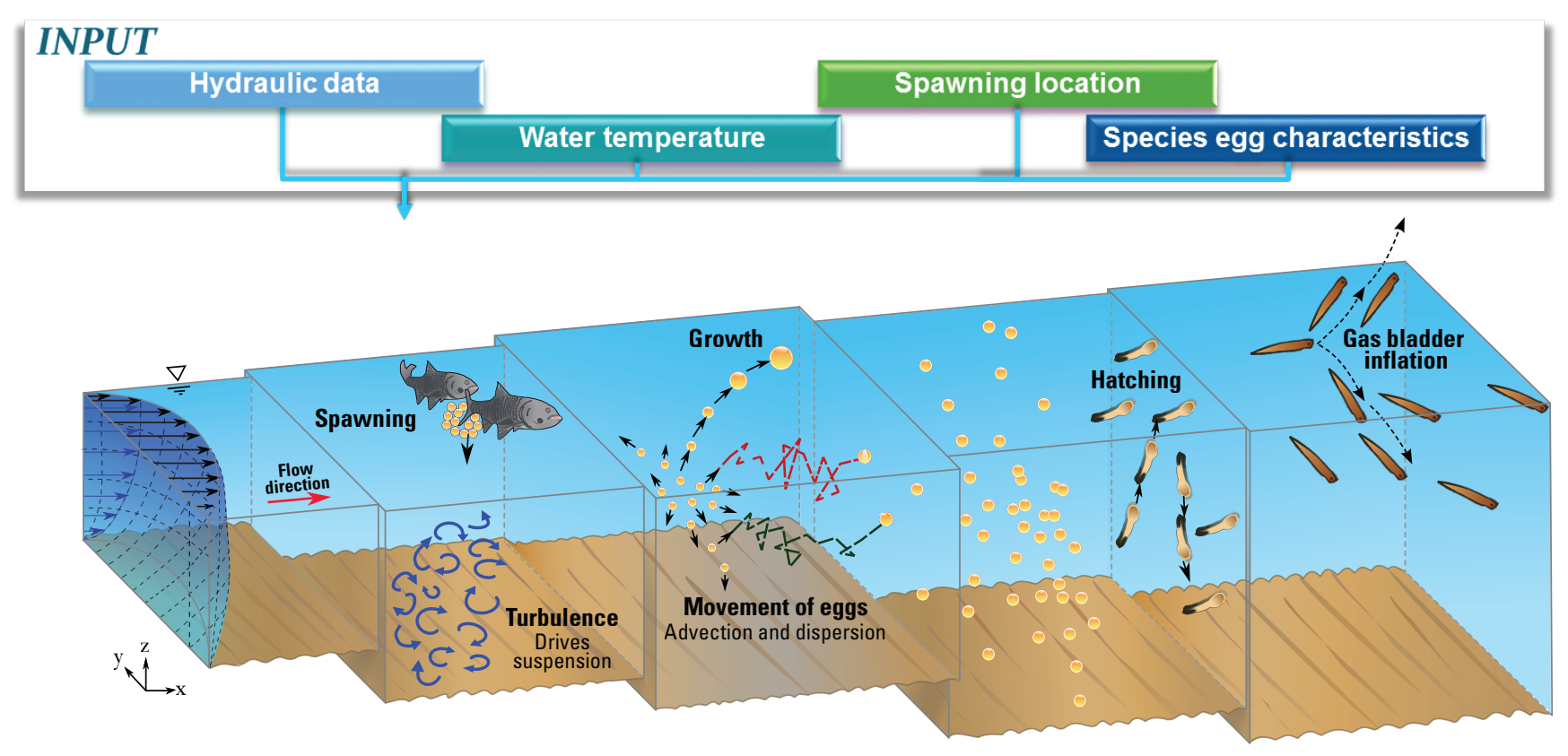

Figure 1. Conceptual schematic of the hydraulic and biologic processes modeled in the Fluvial Egg Drift Simulator (FluEgg; adapted from Garcia and others, 2013).

where
$h \quad$ is the total flow depth, in meters;
$\kappa \quad$ is the von Kármán constant $(0.41)$, unitless;
$u_{*} \quad$ is the shear velocity, in meters per second; and
$U \quad$ is the average velocity, in meters per second, for the cell (Keulegan, 1938).

The lateral variation in streamwise velocity is estimated using the beta probability density function (Seo and Baek, 2004):

$$
u\left(y^{\prime}\right)=u(z) \frac{\Gamma(\alpha+\beta)}{\Gamma(\alpha) \Gamma(\beta)}\left(y^{\prime}\right)^{\alpha-1}\left(1-y^{\prime}\right)^{\beta-1}, 0<y^{\prime}<1
$$

where

$u\left(y^{\prime}\right) \quad$ is the longitudinal velocity, in meters per second, at the fractional lateral position $y^{\prime}=\frac{y}{W}$, where $W$, in meters, is the total width of the channel;

$u(z) \quad$ is the longitudinal velocity for a height $z$, in meters, above the bed (calculated using the logarithmic velocity profile);

$\Gamma \quad$ refers to a gamma function; and

$\alpha$ and $\beta \quad$ are parameters that affect the shape of the distribution, unitless.

In FluEgg, $\alpha$ and $\beta$ are both set equal to 1.85 (Seo and Baek, 2004), giving a symmetrical distribution with the highest velocity at the center of the channel. Modeling the variation in longitudinal velocity along lateral and vertical profiles allows for the effects of shear dispersion to be approximated.

The biological development of the eggs is described by functions that model the changes in egg density and diameter over time and as a function of water temperature as the eggs develop (Garcia and others, 2013, 2015; George and others, 2017). The time at which the eggs hatch into larvae is calculated as a function of water temperature (Chapman and George, 2011; Murphy and Jackson, 2013; George and Chapman, 2013, 2015). When the larvae hatch from the eggs, they have the ability to swim vertically and have been observed to undulate over the full water column in both stationary and flowing water in a laboratory setting (Chapman and George, 2011; George and Chapman, 2013, 2015; Prada and others, 2018). Larvae that undulate over the water column sample the full vertical profile of velocity, so for simplicity the larvae are simulated as neutrally buoyant (Murphy and others, 2016). The time at which the larvae reach the gas bladder inflation (GBI) stage is also a function of water temperature 
(Chapman and George, 2011; George and Chapman, 2013, 2015). At the GBI stage, the larvae develop the ability to swim horizontally and begin to leave the drift in search of off-channel nursery habitat (Chapman and George, 2011). Therefore, FluEgg is not appropriate to use for simulating larval transport beyond the GBI stage.

Each egg/larva is individually transported through each cell of the model domain according to the hydraulic conditions at the egg/larva's location at each time step. The movement of each egg/larva in the longitudinal and lateral directions is simulated with an advective component, in which the egg is assumed to move at the same velocity as the water, and a diffusive component, which is estimated using a random-walk model (Garcia and others, 2013). The vertical movements of eggs/larvae are also simulated with advective and diffusive components but include an additional component to account for egg settling. The settling velocity for larvae is assumed to be zero. The egg/larvae transport model does not fully include the effects of drag, lift, added mass, or Basset forces, though these are expected to be somewhat small in this context. The water surface, channel bed, and channel banks are simulated as reflective boundaries and therefore do not capture the possibility that an egg/larva may be deposited on the bed for an extended period of time. The transport model can be run in reverse, though several caveats apply and are described in more detail in the "Reverse Modeling" section. A more detailed description of the biological development models and the transport and dispersion models within FluEgg can be found in Garcia and others $(2013,2015)$ and Murphy and others (2016).

There are several limitations to using a simple rectangular channel geometry and $1 \mathrm{D}$ hydraulics to characterize the $3 \mathrm{D}$ channel geometries and flow fields present in natural river channels. Simple models for the lateral and vertical profiles of longitudinal velocity are applied, but in general, FluEgg cannot account for complex hydrodynamic effects. FluEgg does not include the effects that channel curvature may have on the velocity distribution in a channel. These can include the generation of secondary flows, lateral flow separation zones, and deviations from the simple lateral and vertical velocity distributions that are constructed in FluEgg. FluEgg does not include the effects of flow steering by bars and bedforms or the effects of flow separation on the lee-side of bedforms or man-made structures. FluEgg is also unable to model the effects of changes in the lateral connectivity of the channel to the flood plain during unsteady flows. All of these effects have the potential to influence the drift of eggs/larvae. In particular, FluEgg cannot describe transient storage effects, such as the potential for eggs to be trapped in low velocity areas like separation zones or to be isolated by the disconnection of floodwater from the main channel. Therefore, caution should be used in applying FluEgg and interpreting results in scenarios with potentially complex hydrodynamics, such as highly sinuous channels, multithreaded channels, channels with complex bed configurations, or channels with man-made structures. In some cases, a two-dimensional or 3D approach may be more appropriate.

\section{Installation}

The executable file of the FluEgg model can be downloaded from the U.S. Geological Survey (USGS) Source Code Archive (https://code.usgs.gov/FluEgg/fluegg/-/releases; Domanski and Berutti, 2020). The model is packaged for running on the Microsoft Windows operating system and x86-64 processor architecture. To run FluEgg, download and save the FluEgg executable file to any directory. The program can run immediately without any installation process by double clicking on the executable file. FluEgg will also create a default results output directory in the same folder in which the executable is stored. If you want to run FluEgg using a model developed with the Hydrologic Engineering Center's River Analysis System (HEC-RAS) as the hydraulic input, you must also have HEC-RAS installed. Downloads and installation instructions for HEC-RAS can be found at https://www.hec.usace.army.mil/software/hec-ras/.

\section{Graphical User Interface for the Fluvial Egg Drift Simulator (FluEgg)}

Start the program by double clicking the FluEgg executable file. This action launches the main graphical user interface (GUI) window for FluEgg (hereafter referred to as the "FluEgg home window"; fig. 2). The FluEgg home window consists of sequential panels to guide users through the various steps required to run a simulation. The FluEgg home window contains three input panels: "Hydraulic Channel," "Eggs," and "Simulation." Additionally, there is an "Open Plot Window" button at the bottom that opens the plotting and data export window. There are seven main steps for running a FluEgg simulation:

1. Load the river input data using either a comma-separated values (CSV) file or a 1D HEC-RAS model (see "The Hydraulic Channel Panel" section).

2. Select the desired eddy diffusivity model (see "Selecting Eddy Diffusivity" section).

3. Fill out the "Eggs" panel (see "The Eggs Panel" section).

4. Fill out the "Simulation" panel (see "The Simulation Panel" section).

5. Select the output location by clicking the "Select Output Folder" button.

6. Run the model by clicking the "Run" button.

7. After the model has run, click "Open Plot Window" to open the plotting and data export window.

There is also an "About" pulldown menu at the top left side of the FluEgg home window. The choices in the menu are "Version," "User Manual," and "Project Page." "Version" 


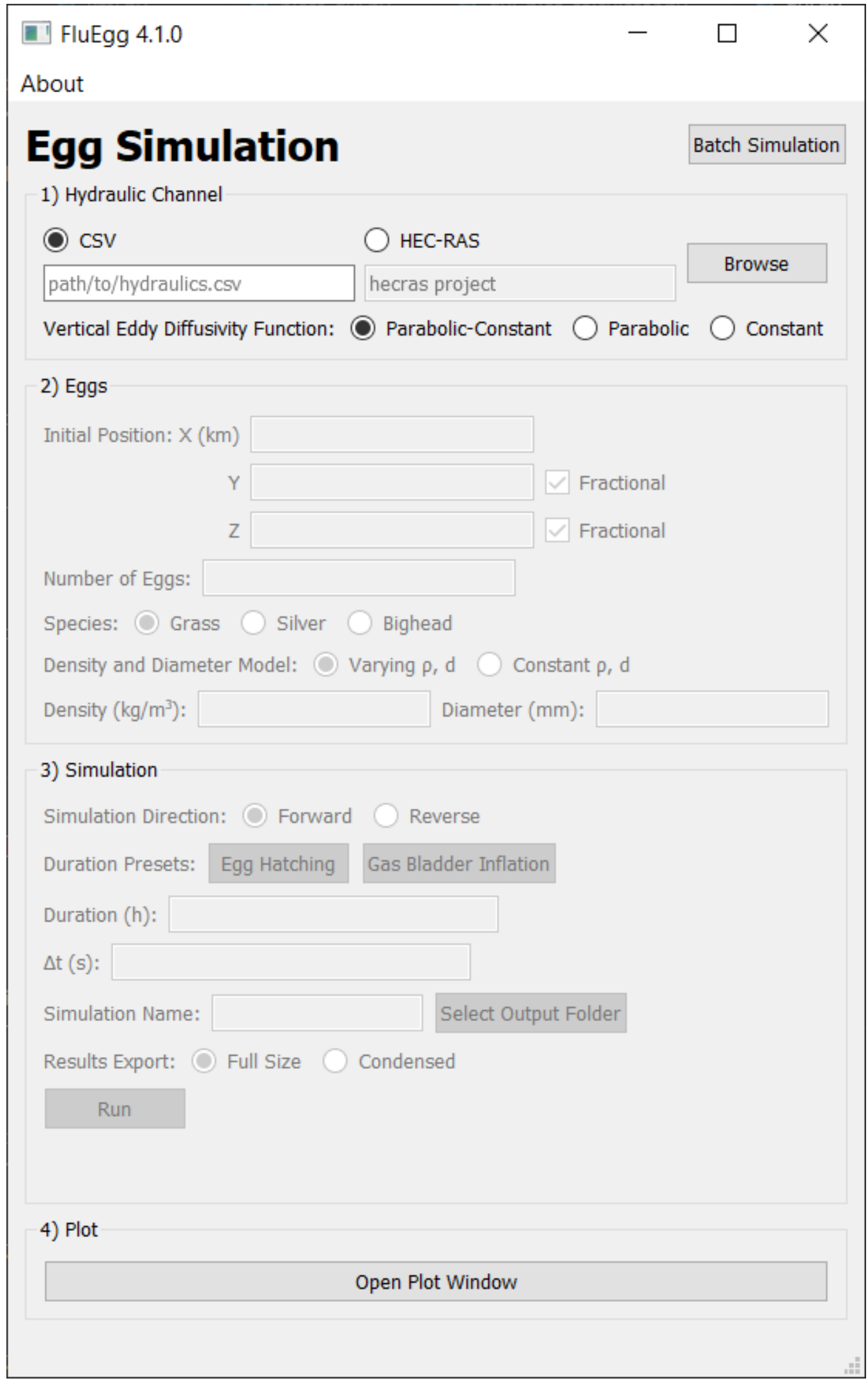

Figure 2. The Fluvial Egg Drift Simulator (FluEgg) home window before loading hydraulic data. 
displays a dialog with version information and "User Manual" and "Project Page" provide links to this user manual and to the official FluEgg web page, respectively.

\section{The Hydraulic Channel Panel}

FluEgg requires the user to supply the hydraulic and environmental conditions for the simulation (referred to herein as the "river input" or "hydraulic channel"). The river input data can be supplied with either a CSV river input file or a HEC-RAS project. Regardless of whether a CSV river input file or a HEC-RAS project is used, the river channel in FluEgg is represented as a series of discrete cells that are rectangular in cross-section and characterized by nine parameters:

1. CellNumber (cell number [unitless])

2. CumlDistance_km (cumulative distance downstream, from the most upstream point in the study reach to the downstream end of the cell [kilometers])

3. Depth_m (average water depth in cell [meters])

4. Q_cms (average water discharge in cell [cubic meters per second])

5. Vmag_mps (average longitudinal velocity in cell [meters per second])

6. Vvert_mps (average vertical velocity in cell [meters per second])

7. Vlat_mps (average lateral velocity in cell [meters per second])

8. Ustar_mps (average shear velocity in cell [meters per second])

9. Temp_C (average water temperature in cell [degrees Celsius])

Note for the average value parameters, estimated values may not be true average values, in which case the hydraulic data would be inaccurate.

\section{Creating a CSV River Input File}

CSV river input files can only be used for steady simulations in which the hydraulic conditions are assumed to not vary in time. A CSV river input file can be as simple or as complex as you choose to make it, depending on the number and size of the cells into which you divide the study reach (cells can have different lengths). Simple CSV river input files can be created using "found" data like navigation maps and gages or using field measurements. If the discharge, average depth, and width are known, the velocity magnitude can be estimated as the discharge divided by the cross-sectional area. The velocity magnitude can also be derived directly from field measurements using hydroacoustic instruments.
There are multiple methods for estimating shear velocity (and the related bed shear stress; see Biron and others, 2004) and different methods will produce different estimates. If velocity has been measured along a vertical profile and the data show a logarithmic profile, the shear stress can be estimated by fitting the data to the equation for a logarithmic velocity profile (eq. 1). If the energy slope $\left(S_{f}\right)$ and hydraulic radius $(R)$, in meters, within each reach are known, the average shear velocity $\left(u_{*}\right)$, in meters per second, for a cell can be computed as $u_{*}=\sqrt{g R S_{f}}$ (Babaeyan-Koopaei and others, 2002), where $g$ is the acceleration due to gravity (9.81 meters per second squared). If the data for other methods are not available, shear velocity can be roughly estimated as 12.5 percent (one-eighth) of the average longitudinal velocity (based on data from Rutherford, 1994). If this simple rough estimate is used, then an analysis to determine how sensitive the FluEgg results are to shear velocity is recommended.

The water temperature can be specified individually for each cell; however, the time to egg hatching and GBI stage will be calculated using the average temperature of all the cells. Note that the hydraulic domain is limited to the bounds of the CSV or HEC-RAS file. The downstream boundary of the FluEgg model domain is infinitely extended to accommodate eggs that leave the downstream boundary of the hydraulic domain. The hydraulic properties of the last cell of the hydraulic domain are used in the transport of eggs and larvae in the extended portion of the model domain.

To create a CSV river input file in Microsoft Excel ${ }^{\circledR}$, use the nine input parameters (listed in the "The Hydraulic Channel Panel" section) as headings in the first row and enter your data in the columns below. Each cell is represented by a new row in the table and should be listed from upstream to downstream. There is no limitation on the number of cells in an input file. Note that the cumulative distance is the total distance from the most upstream point in the overall study reach of the river (the upstream boundary of the FluEgg model domain) to the downstream end of the cell and is not the distance from the previous cell (or cell length). The cumulative distance is also not necessarily the distance from the proposed spawning location as defined in the "Eggs" panel in FluEgg. An example of a simple river input file prepared with Microsoft Excel ${ }^{\circledR}$ is shown in figure 3 .

\section{Preparing a HEC-RAS Project for Use in FluEgg}

The only way to run unsteady (time-varying) hydraulic conditions in FluEgg is to use a 1D HEC-RAS model. HEC-RAS models can also be used for steady flow FluEgg simulations. However, if a steady model is used, consider creating a CSV river input file, because the CSV river input file loads more quickly than a HEC-RAS project, is easier to share with collaborators, and allows water temperature to vary between cells. Currently, it is not possible to vary the water temperature in space or time when using a HEC-RAS project file with FluEgg. If you plan to use a HEC-RAS project, the 


\begin{tabular}{|r|r|r|r|r|r|r|r|r|r|r|}
\hline \multicolumn{1}{|c|}{ A } & B & \multicolumn{1}{c}{ C } & \multicolumn{1}{c}{ D } & \multicolumn{1}{c|}{ E } & F & G & H & I \\
\hline 1 & CellNumber & CumlDistance_km & Depth_m & Q_cms & Vmag_mps & Vvert_mps & Vlat_mps & Ustar_mps & Temp_C \\
\hline 2 & 1 & 20 & 5 & 2832 & 1.72 & 0 & 0 & 0.2150 & 22 \\
\hline 3 & 2 & 40 & 5 & 2832 & 1.67 & 0 & 0 & 0.2088 & 22 \\
\hline 4 & 3 & 60 & 5 & 2832 & 1.57 & 0 & 0 & 0.1963 & 22 \\
\hline 5 & 4 & 5 & 2832 & 1.89 & 0 & 0 & 0.2363 & 22 \\
\hline 6 & 5 & 100 & 5 & 2832 & 1.62 & 0 & 0 & 0.2025 & 22 \\
\hline
\end{tabular}

Figure 3. A simple example of a river input file with five cells.

HEC-RAS model must first be run within HEC-RAS for the steady flow(s) or unsteady hydrograph(s) of interest. FluEgg will extract the necessary hydraulic data from the HEC-RAS project files for the specified simulation of interest.

\section{Loading the Hydraulic Channel}

In the "Hydraulic Channel" panel, the user specifies the hydrodynamic and environmental characteristics for each individual cell. These data may be loaded from two different sources: CSV files and HEC-RAS projects (the HEC-RAS version 5.0.7 or 5.0.3 program will need to be installed on your computer to access this option). First, use the radio buttons in the "Hydraulic Channel" panel to select "CSV" or "HEC-RAS," then click the "Browse" button (fig. 2). Navigate to the desired file, select it, then click "Open." Note that if you chose "CSV" in the FluEgg home window you will only be able to see files with a .csv extension, and if you chose "HEC-RAS" you will only be able to see files with a .prj extension.

If you selected a CSV river input file, there is no additional entry needed for the hydraulic channel. In contrast, if a HEC-RAS project is selected, an additional "HEC-RAS Settings" dialog will open (fig. 4). In this dialog, select whether the project is steady or unsteady and input the temperature. Select the "Plan" and in the case of steady runs, the "Profile." For unsteady runs, the start time must be entered as well. The start time is the time at which spawning is initiated in the FluEgg simulation (in the same time zone as the HEC-RAS model). FluEgg assumes that eggs are fertilized immediately after spawning. Click "Ok" to return to the FluEgg home window.

\section{Selecting Eddy Diffusivity}

Once you have returned to the FluEgg home window, the rest of the panels will become editable. You can specify an eddy diffusivity function using the radio buttons at the bottom of the "Hydraulic Channel" panel (figs. 2, 5). The vertical eddy diffusivity function describes the vertical diffusion of fluid momentum. There are three predetermined profile functions for the calculation of the fluid eddy viscosity: parabolic-constant turbulent diffusivity (the default), parabolic turbulent diffusivity, and constant turbulent diffusivity (Garcia and others, 2013). The default setting is suitable for most applications in natural rivers. For more information on the differences between the eddy viscosity options, refer to Garcia and others (2013).

\section{The Eggs Panel}

The input parameters required in the "Eggs" panel of the FluEgg home window are the 3D "Initial Position" (X, Y, Z), the number of eggs to be released in the spawning event, the species of the eggs, and the model for egg density and diameter (fig. 5). It is recommended that you determine the minimum number of eggs required to give statistically

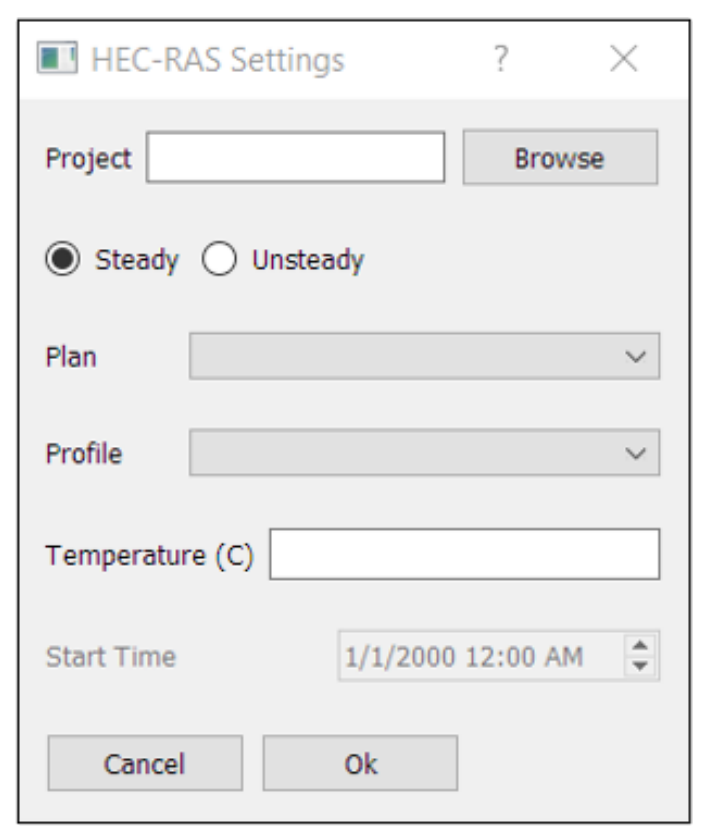

Figure 4. The Hydrologic Engineering Center's River Analysis System (HEC-RAS) settings subinterface with the Fluvial Egg Drift Simulator (FluEgg). 


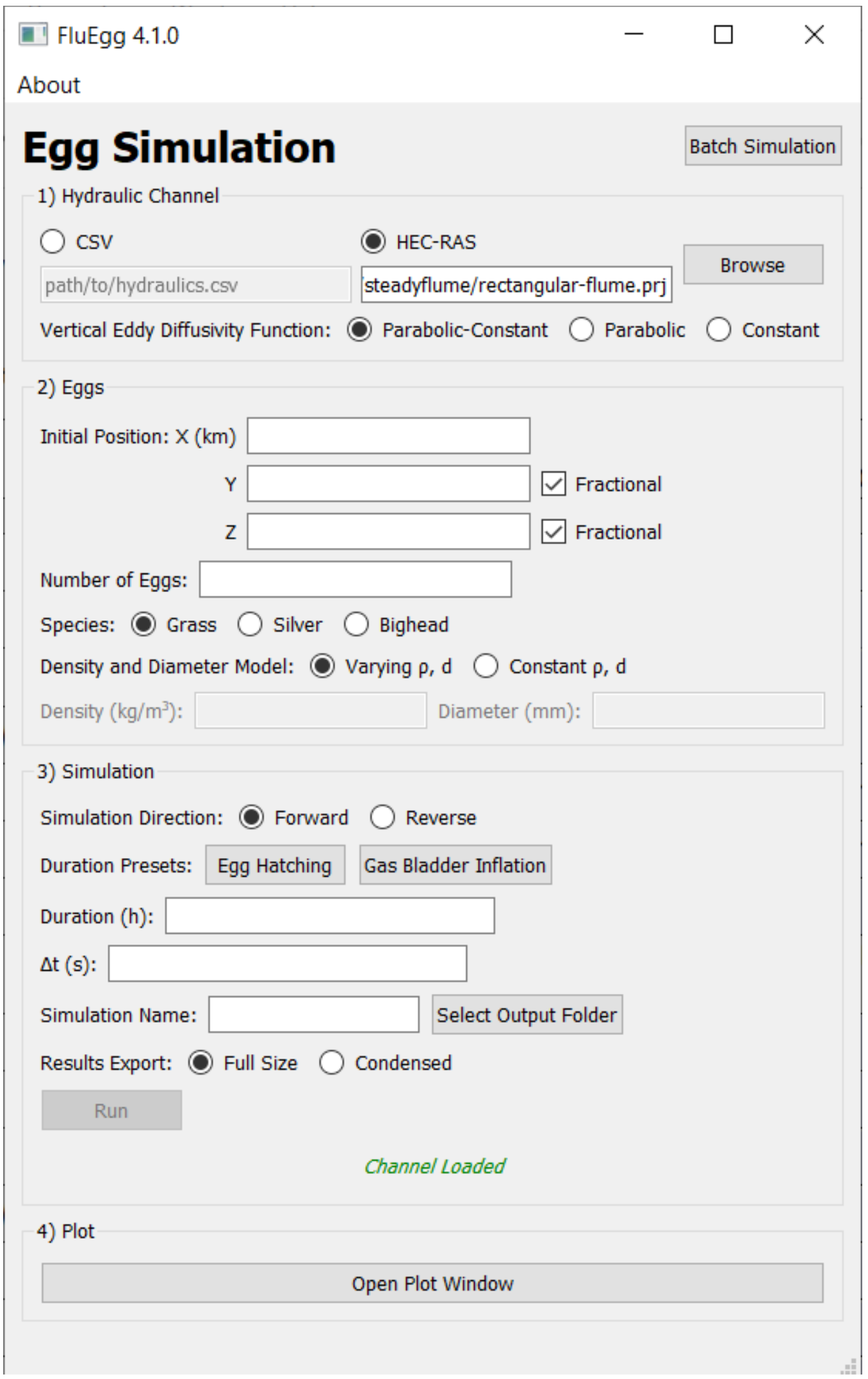

Figure 5. The Fluvial Egg Drift Simulator (FluEgg) home window after loading river input parameters. 
significant results for a given scenario. This determination can be made by running repeat simulations for increasingly large numbers of eggs until the results are no longer dependent on the number of eggs (within a given tolerance margin). This point will change depending on the various simulation parameters, but generally, a value of at least 5,000 eggs would be a good starting point. More information about this process can be found in the appendix of Garcia and others (2013).

The 3D initial position of the eggs represents the spawning location for "forward" simulations, which may be a known spawning location or a hypothesized spawning location depending on the availability of data for a particular stream. For "reverse" simulations (see "Reverse Modeling" section), the initial position of the eggs represents the location where the eggs/larvae were sampled. In this coordinate system, X represents the longitudinal (streamwise) direction, $\mathrm{Y}$ represents the lateral (cross-stream) direction, and $\mathrm{Z}$ represents the vertical direction (fig. 6). The longitudinal spawning location $(\mathrm{X})$ is entered as the distance in kilometers from the most upstream point of the study reach (the upstream boundary of the model domain). $\mathrm{Y}$ and $\mathrm{Z}$ can either be entered in meters or, if the fractional box is checked, as a fractional distance relative to total width or depth (at the longitudinal spawning location, $\mathrm{X}$ ). The $\mathrm{Y}$ and $\mathrm{Z}$ axes are defined differently depending on whether the initial position is given as a fractional distance or in meters (fig. 5). If fractional distances are used for $\mathrm{Y}$, a fractional value of $\mathrm{Y}=0$ represents the right bank, $\mathrm{Y}=0.5$ is the midpoint, and $\mathrm{Y}=1$ represents the left bank, looking downstream. If $\mathrm{Y}$ is entered in meters, $\mathrm{Y}=0$ is located at the midpoint of the channel and $\mathrm{Y}$ is positive toward the left bank and negative toward the right bank, looking downstream. If fractional distances are used for $Z$, the channel bed is located at $Z=0$ and $Z$ increases with height above the bed, such that $Z=1$ represents the water surface. In general, fractional distances are input as decimal numbers from 0 to 1 , inclusive. If $Z$ is entered in meters, $Z=0$ is located at the water surface and depths below the water surface are given as negative numbers, such that the channel bed is located at $\mathrm{Z}=-\mathrm{H}$, where $\mathrm{H}$ is the water depth (at the longitudinal spawning location, $\mathrm{X}$ ).

You can choose from three invasive carp species to use as a model for the simulation: grass carp, silver carp, and bighead carp. You can then select the model for the egg diameter and density. There are two choices: "Varying $\rho$, d" to use the time series of egg diameter (d) and density ( $\rho$ ), and "Constant $\rho$, d" to use a user-specified constant egg diameter and density (fig. 5). If "Varying $\rho, d$ " is selected, the egg density and diameter time series for the selected invasive carp species will be computed and used (George and others, 2017). If the "Constant $\rho, d$ " option is selected, the diameter and density fields will become editable, and you must specify values for these fields before you run a simulation (fig. 7). This option is useful for simulating eggs of other pelagic-spawning species or generic particles (for example, plastics).

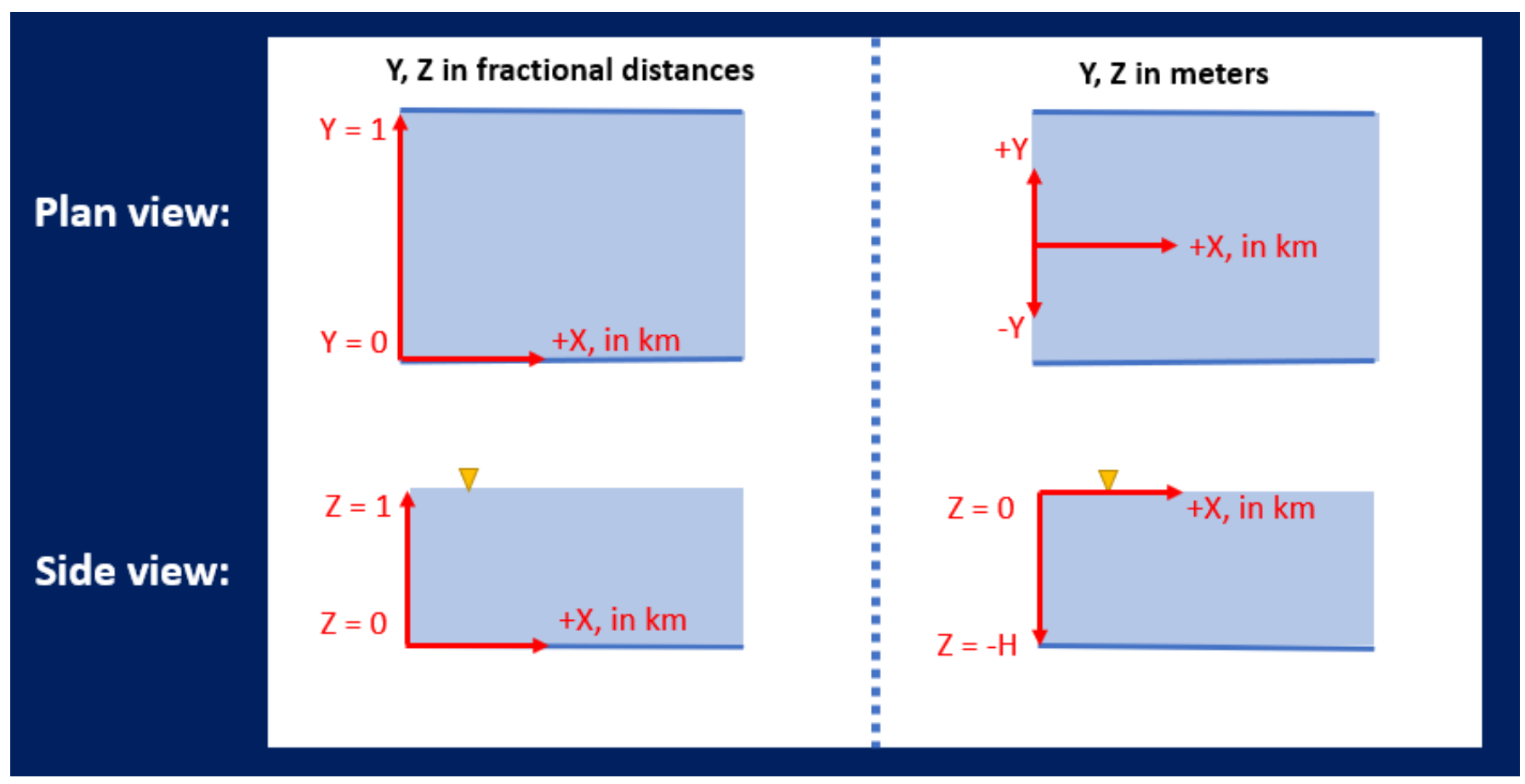

Figure 6. The Fluvial Egg Drift Simulator (FluEgg) coordinate system when $Y$ and $Z$ are fractional distances (left panel) or entered in meters (right panel). In the bottom right panel, $\mathrm{H}$ is the water depth and $\mathrm{Z}=-\mathrm{H}$ refers to the channel bed. The yellow triangle indicates the water surface. 


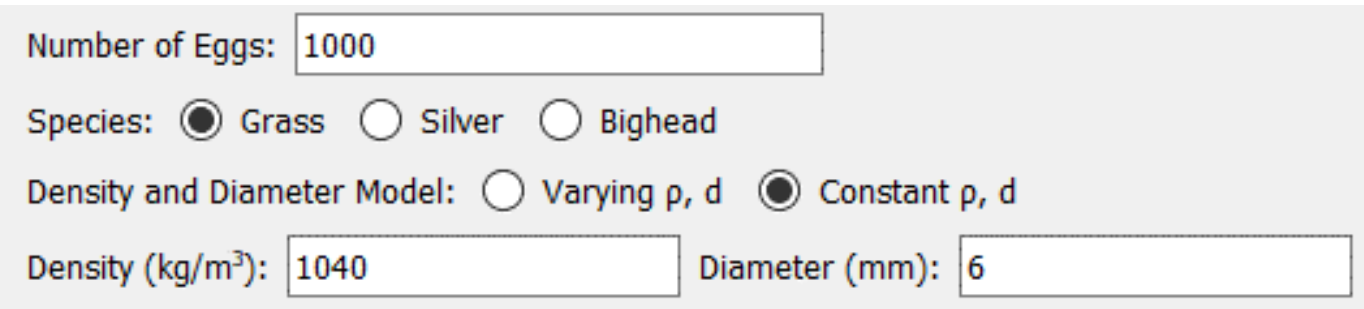

Figure 7. The "Egg" characteristics panel within the Fluvial Egg Drift Simulator (FluEgg) if the user selects the "Constant $\rho, d$ " option.

\section{The Simulation Panel}

In the "Simulation" panel, specify the simulation direction, the duration, the time step $(\Delta \mathrm{t})$ size, and the simulation name (fig. 5). The simulation direction indicates whether the model will be run forward or reverse in time. Forward modeling estimates how a plume of eggs/larvae drifts downstream from a user-specified spawning location. Reverse modeling is used to back-calculate possible spawning locations based on information that can be gathered from field samples of eggs/larvae. See the "Reverse Modeling" section for more information.

The simulation duration is the length of the simulation in hours. Clicking the preset buttons below the simulation direction selection ("Egg Hatching" or "Gas Bladder Inflation") will automatically populate the duration field with the time to hatching or the time to the GBI stage based on the species and the water temperature. The simulation duration can be set to any amount of time but use caution in interpreting results for simulations that exceed the hatching time in duration. After hatching, the larvae can swim vertically but are simply simulated in FluEgg as neutrally buoyant particles. At the GBI stage, the larvae develop the ability to swim horizontally and cannot be simulated as passively drifting particles (Chapman and George, 2011); therefore, running FluEgg past the GBI stage is not appropriate.

The simulation time step $(\Delta t)$ is entered in seconds and must fulfill the appropriate random-walk displacement stability criteria established by Garcia and others (2013). The largest allowable time step to meet the criteria depends on the selected species, water temperature, shear velocity, and water depth. If you select a time step that is too large to ensure the appropriate criteria is satisfied, upon running the program, the simulation will fail and a warning message will appear, indicating the simulation's calculated maximum time step (fig. 8). Choosing a time step slightly smaller than the maximum calculated time step should satisfy the criteria and allow the simulation to proceed, but it might take multiple tries to satisfy the criteria. Therefore, the user can make an initial guess for the time step when filling out the "Simulation" setup panel and reduce it if necessary. In most cases, a time step of less than 30 seconds will be required.

The "Simulation Name" entered by the user will be used as the name for the results file. By default, the results are saved in a "results" folder in the same directory as the FluEgg executable. To save the results in another location, click the "Select Output Folder" button and choose the desired directory. FluEgg will overwrite a file with the same name in the same folder, so be careful not to duplicate names within the same folder. Finally, select whether the exported results should be "Full Size" or "Condensed." For full size results, the 3D position of each egg/larva at each time step is saved into the exported results file. In the condensed results file, the plume of eggs/larvae is divided into 101 equally spaced quantiles and the quantile positions are exported. Plotting and many calculations will be virtually identical for the full size and condensed results; however, full size results are suggested if additional custom calculations are required.

After confirming all required input parameters have been entered and are acceptable, the simulation is ready to run. To run the simulation, click the "Run" button. You will not be able to
Figure 8. The warning message generated by the Fluvial Egg Drift Simulator (FluEgg) informing the user that a smaller time step must be used in the simulation.

\section{OK}

\section{The following error message was reported: Time step size is greater than maximum time step of 3.5235672411081396}


click this button if any of the required input fields are empty. Informational text and a progress bar will appear below the "Run" button, detailing the progress of the simulation (note that the last 10 percent of the simulation on the progress bar can take substantially longer than the first 90 percent). Once complete, the text will display " $<$ Filename $>$ saved." The results will automatically be saved as a $\mathrm{h} 5$ file in either the default results folder or the folder specified by the user. If you click on the "Open Plot Window" button after completing a simulation (fig. 9), the results from that simulation will be automatically loaded into the plotting and data export window. You can also click the "Open Plot Window" button and load an existing .h5 results file for plotting and analysis. See the "Plotting and PostProcessing Results" section for more details.

\section{Batch Simulations}

To perform multiple FluEgg runs, click the "Batch Simulation" button on the top right corner of the home window (fig. 2). This will open a window designed for creating a batch of simulation runs (fig. 10). The major difference between the home and batch windows is the "Iterate" checkboxes, which are present in the batch window.

To use the batch simulation window, work through the same steps as the single simulation window. If you want to vary an input throughout the batch process (for instance, the initial X, Y, or Z position), click the "Iterate" checkbox to the right. Some additional input boxes will become editable. In these boxes, input the starting value, the ending value, and how many steps to iterate (fig. 11). For example, if you choose to iterate $\mathrm{X}$ and set the starting value to 0 , the ending value to 100 , and the number of steps to 3 , then your batch simulation will consist of three runs with $X=0, X=50$, and $X=100$ (all other settings held constant).

The three vertical eddy diffusivity functions and the three invasive carp species can also be iterated. Additionally, if a HEC-RAS hydraulic model is used, water temperature may be an iterated parameter. Click on the checkbox next to "Overwrite Temperature" in the "Hydraulic Channel" panel to use this functionality (fig. 10).

Any number of variables may be iterated for the same batch process; however, the number of simulations the batch process runs is the number of all possible combinations of these iterated variables. To calculate the number of simulations in the batch, multiply the number of steps for each variable together. For example, in figure 11 there are 6 steps for $Y, 11$ steps for $Z$, and the user has chosen to iterate the three species. Therefore, the number of simulations would be 6 multiplied by 11, then multiplied by 3 , which gives 198 .

The simulation parameters for batch runs are the same as they would be for a single run, with the exception of the duration presets (fig. 10). Unlike the single simulation window, which uses push buttons for the hatching and GBI time presets, the batch simulation window uses radio buttons. Selecting either the "Egg Hatching" or "Gas Bladder Inflation" radio button will calculate the corresponding duration preset for each individual simulation in the batch based on the species and temperature specified for the simulation. These duration presets cannot be modified. Alternatively, the user can select the "Manual" radio button to specify a single duration that will be used for all simulations in the batch. You must also enter the "Batch Name" and select a full size or condensed output file in the "Batch Settings" panel. Once ready, click on the "Run" button. FluEgg will create a new folder named with the "Batch Name" and store the .h5 files for all the runs there, along with a text file named "_batch_.txt" with information about the batch settings (fig. 12). Each individual run will be named with the "Batch Name" and the value(s) of the variable(s) that were iterated.

\section{Reverse Modeling}

Reverse modeling in the FluEgg program is enabled by selecting "Reverse" in the "Simulation" section in the simulation window (fig. 5). The reverse modeling feature runs the biological and hydrodynamic models backwards in time from a known egg/larval sampling location using a reverse-time particle tracking method (Zhu and others, 2018). This feature is useful, for example, if eggs/larvae are collected in a river and the researcher wants to determine where the eggs/larvae were likely spawned. The information needed for a reverse simulation includes the location and time that an egg/larva was collected, the water temperature, and the age of the egg/larva in hours post-fertilization as determined by developmental stage and water temperature (see Embke and others [2019] for a grass carp example). Using an unsteady HEC-RAS model, rather than a steady-state model or CSV river input file, is strongly recommended for reverse simulations.

It is not possible to reverse the dispersion that occurs as eggs drift downstream. Therefore, a reverse simulation does not provide an exact back-calculation of a spawning location. Rather, a distribution of possible spawning locations is generated by simulating a large number of eggs and including the random-walk component in the reverse particle transport model. The "most likely" spawning location can be identified from this distribution.

Running a reverse FluEgg simulation is very similar to running a forward simulation. There are three key differences. First, the initial position $(\mathrm{X}, \mathrm{Y}, \mathrm{Z})$ in the "Eggs" panel will represent the sampling location (where the egg/larva was collected from the river), as opposed to the spawning location. Second, the simulation time (the amount of time the eggs will be tracked upstream in the model domain) should be set equal to the age of eggs (in decimal hours) at the time they were collected from the river. Third, the start time (entered in the HEC-RAS window; fig. 4) should be set to the time that the egg/larva was sampled from the river. A large number of eggs should be used, similar to a forward simulation, even though you may only have sampling data for a small number of eggs/larvae. Larval samples at developmental stages beyond the GBI stage cannot be used for reverse modeling. 


\section{About}

\section{Egg Simulation}

Batch Simulation

1) Hydraulic Channel

path/to/hydraulics.csv

(2) HEC-RAS steadyflume/rectangular-flume.prj

Browse

(a)

Vertical Eddy Diffusivity Function: $\bigcirc$ Parabolic-Constant $\bigcirc$ Parabolic $\bigcirc$ constant

2) Eggs

Initial Position: $\mathrm{X}(\mathrm{km}) 0$

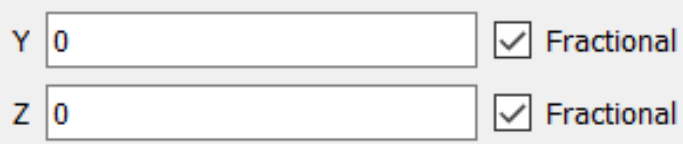

Number of Eggs: 1000

Species: $\bigcirc$ Grass $\bigcirc$ silver $\bigcirc$ Bighead

Density and Diameter Model: $\bigcirc$ Varying $p, d \quad$ Constant $p, d$

Density $\left(\mathrm{kg} / \mathrm{m}^{3}\right): 1040 \quad$ Diameter $(\mathrm{mm}): 6$

3) Simulation

Simulation Direction: 9 Forward $\bigcirc$ Reverse

Duration Presets: Egg Hatching Gas Bladder Inflation

Duration (h): 17

$\Delta \mathrm{t}(\mathrm{s}): 10$

Simulation Name: rectangular flume Select Output Folder

Results Export: 9 Full Size Condensed

Run

rectangular flume saved.

4) Plot

\section{Open Plot Window}

Figure 9. The Fluvial Egg Drift Simulator (FluEgg) home window after a successful simulation. 
FluEgg 4.1.0

About

\section{Batch Simulation}

1) Hydraulic Channel

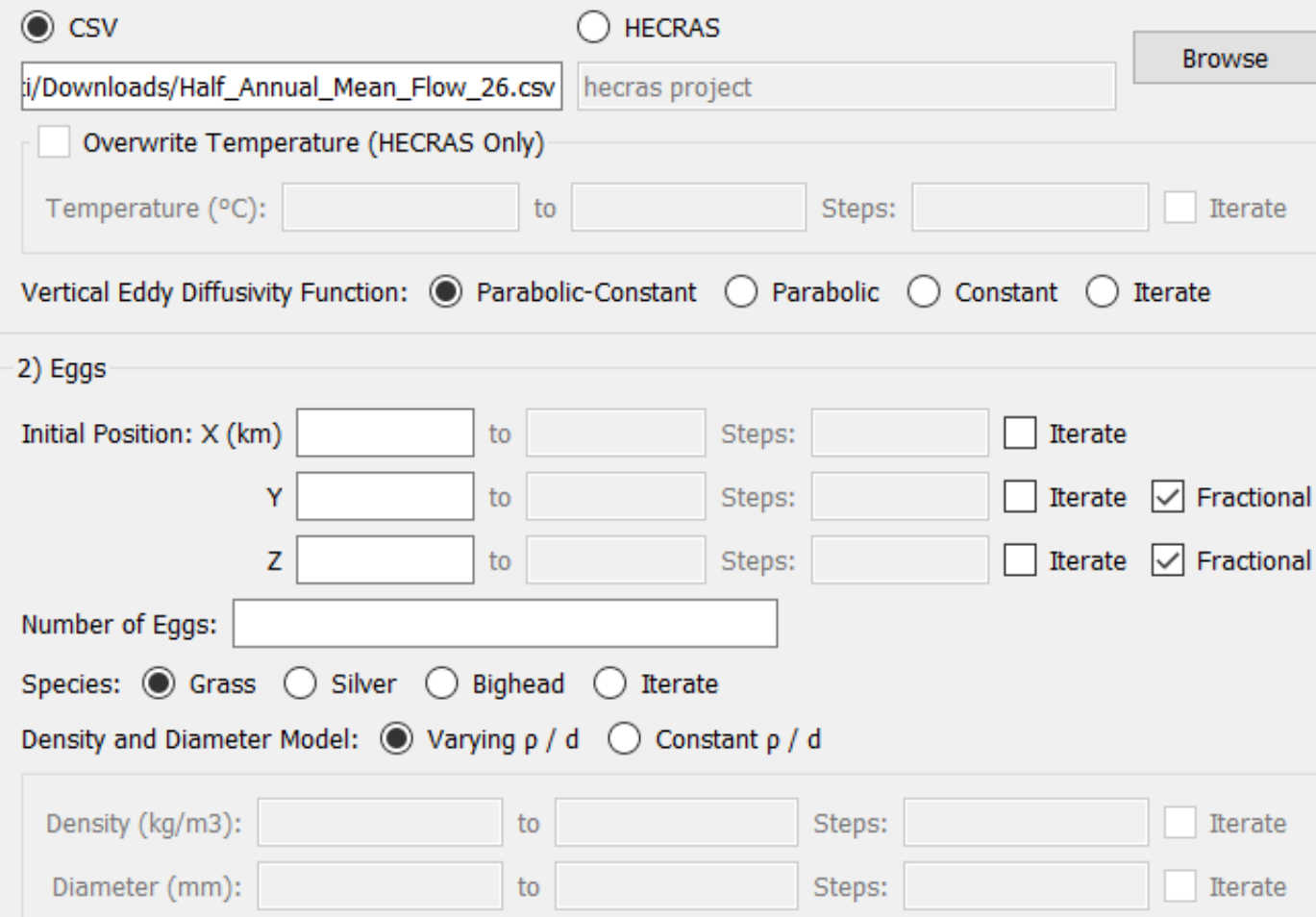

3) Simulation

Simulation Direction: $\bigcirc$ Forward $\bigcirc$ Reverse

Duration Presets: $\bigcirc$ Manual $\bigcirc$ Egg Hatching $\bigcirc$ Gas Bladder Inflation

Duration (h):

$\Delta \mathrm{t}(\mathrm{s})$ :

4) Batch Settings

Batch Name: $\square$ Select Output Folder

Results Export: $\bigcirc$ Full Size $\bigcirc$ condensed

Run

Channel Loaded

5) Plot

Open Plot Window

Figure 10. The batch simulation window within the Fluvial Egg Drift Simulator (FluEgg). 


\section{2) Eggs}

\begin{tabular}{|c|c|c|c|c|c|c|}
\hline Initial Position: X (km) & 25 & to & & Steps: & & $\square$ Iterate \\
\hline $\mathrm{Y}$ & 0 & to & 1 & Steps: & 6 & $\checkmark$ Iterate \\
\hline Z & 0 & to & 1 & Steps: & 11 & $\checkmark$ Iterate \\
\hline
\end{tabular}

Number of Eggs: 1000

Species: $\bigcirc$ grass $\bigcirc$ silver $\bigcirc$ Bighead $\bigcirc$ Iterate

Density and Diameter Model: Varying $\rho / d \quad \bigcirc$ Constant $\rho / d$

Density $(\mathrm{kg} / \mathrm{m} 3): \square$ Steps: $\square$ to $\square$ to $\square$ Steps: $\square$ Iterate
Diameter (mm): $\square$ Iterate

Figure 11. An example batch simulation setup within the Fluvial Egg Drift Simulator (FluEgg).

Name

算_batch_

Unsteady Flume $\mathrm{z}=0.00$ bighead.h5

Unsteady Flume $\mathrm{z}=0.00$ grass.h5

Unsteady Flume $\mathrm{z}=0.00$ silver.h5

Unsteady Flume $\mathrm{z}=0.03$ bighead.h5

Unsteady Flume $\mathrm{z}=0.03$ grass.h5

Unsteady Flume $\mathrm{z}=0.03$ silver.h5

Unsteady Flume $\mathrm{z}=0.06$ bighead.h5

Unsteady Flume $\mathrm{z}=0.06$ grass.h5

Unsteady Flume $\mathrm{z}=0.06$ silver.h5 \begin{tabular}{l|l|l} 
Date modified & Type & Size
\end{tabular}

6/9/2020 12:52 PM Text Document 1 KB

6/9/2020 2:04 PM H5 File 1,688,094 KB

6/9/2020 2:04 PM H5 File 1,688,094 KB

6/9/2020 2:03 PM H5 File 1,688,094 KB

6/9/2020 2:04 PM H5 File 1,688,094 KB

6/9/2020 2:04 PM H5 File 1,688,094 KB

6/9/2020 2:05 PM H5 File 1,688,094 KB

6/9/2020 2:04 PM H5 File 1,688,094 KB

6/9/2020 2:03 PM H5 File 1,688,094 KB

6/9/2020 2:05 PM H5 File 1,688,094 KB

Figure 12. An example of the batch simulation output within the Fluvial Egg Drift Simulator (FluEgg). 
Reverse FluEgg simulations result in a distribution of potential spawning locations, which can be plotted with the longitudinal distribution plot and the location quantile plots that are described in the "Plotting and Post-Processing Results" section. In the example shown in figure 13, the egg sampling location (initial position) is $20 \mathrm{~km}$ downstream from the upstream model boundary, and the age of the eggs (reverse simulation duration) is 15 hours. The results show that the spawning location of the eggs could span from approximately 13 to $19 \mathrm{~km}$ downstream from the upstream boundary of the model, with a median spawning location of about $17 \mathrm{~km}$ and greatest fraction of eggs at $17.5 \mathrm{~km}$.

\section{Plotting and Post-Processing Results}

Once you set up and successfully run the model, click the "Open Plot Window" button at the bottom of the FluEgg home window (fig. 9). Upon clicking the button, a new interface will appear that contains the list of plotting and post-processing tools (fig. 14). This interface provides options for plotting and exporting FluEgg model results. Please note that once the plotting interface has been opened, the user cannot return to the FluEgg home window.

If a simulation was run immediately prior to opening the plot window, then the results will automatically be loaded. If you want to analyze a different dataset, click on the "Load File" button on the top right and navigate to the desired .h5 file. After the file is loaded, a summary of the results file will appear underneath the displayed file path, displaying number of eggs, species, duration, and time step. Large files may take a minute or two to load, especially if accessing files on a remote server or shared drive.

Distribution of Egg Locations Along the X-Axis at $t=15.0 \mathrm{~h}$

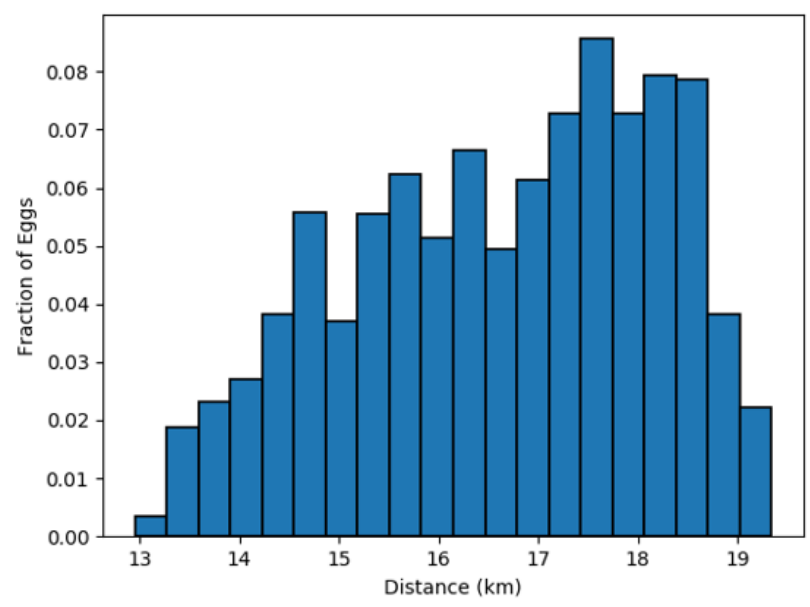

Plotting and post-processing tools are organized into three groups, which are accessed by three tabs: "At a location," "At a time," and "Miscellaneous" (fig. 14). Each tab contains multiple plotting and post-processing options. As you move between tabs and select different plotting and postprocessing options, the settings on the right one-half of the window will adjust to allow you to edit only what is required for that particular plot. There are two Keyhole Markup Language (KML)-generating utilities in the plotting options: one in the "At a time" tab and one in the "Miscellaneous" tab. Before using a KML-generating utility, you need to have or create a KML file of the river centerline. FluEgg uses the centerline to transform the streamwise coordinates used in FluEgg into real-world latitude and longitude coordinates. The centerline can be generated in Google Earth ${ }^{\mathrm{TM}}$ or in another geographic information system software as a polyline feature and exported as a KML file. The river centerline needs to be digitized from upstream to downstream and the upstream end of the centerline must be at the $X=0$ position (that is, the upstream end of the KML river centerline must match the upstream end of the hydraulic channel used in the FluEgg simulation). It is highly recommended to use the same linear reference used to construct the CSV input file or the centerline from the HEC-RAS model, which will ensure the input and output stationing are consistent.

\section{"At a location" Plots}

The "At a location" tab provides options to plot and export information about how many eggs/larvae are present at or have passed a particular location over time (fig. 14). These plots are useful if you want to look at the results as if you were an observer standing on the bank at a location downstream

Figure 13. Two useful plots for identifying potential spawning locations using output from the Fluvial Egg Drift Simulator (FluEgg). The left panel shows an example of the longitudinal distribution plot and the right panel shows an example of the location quantile plot. 


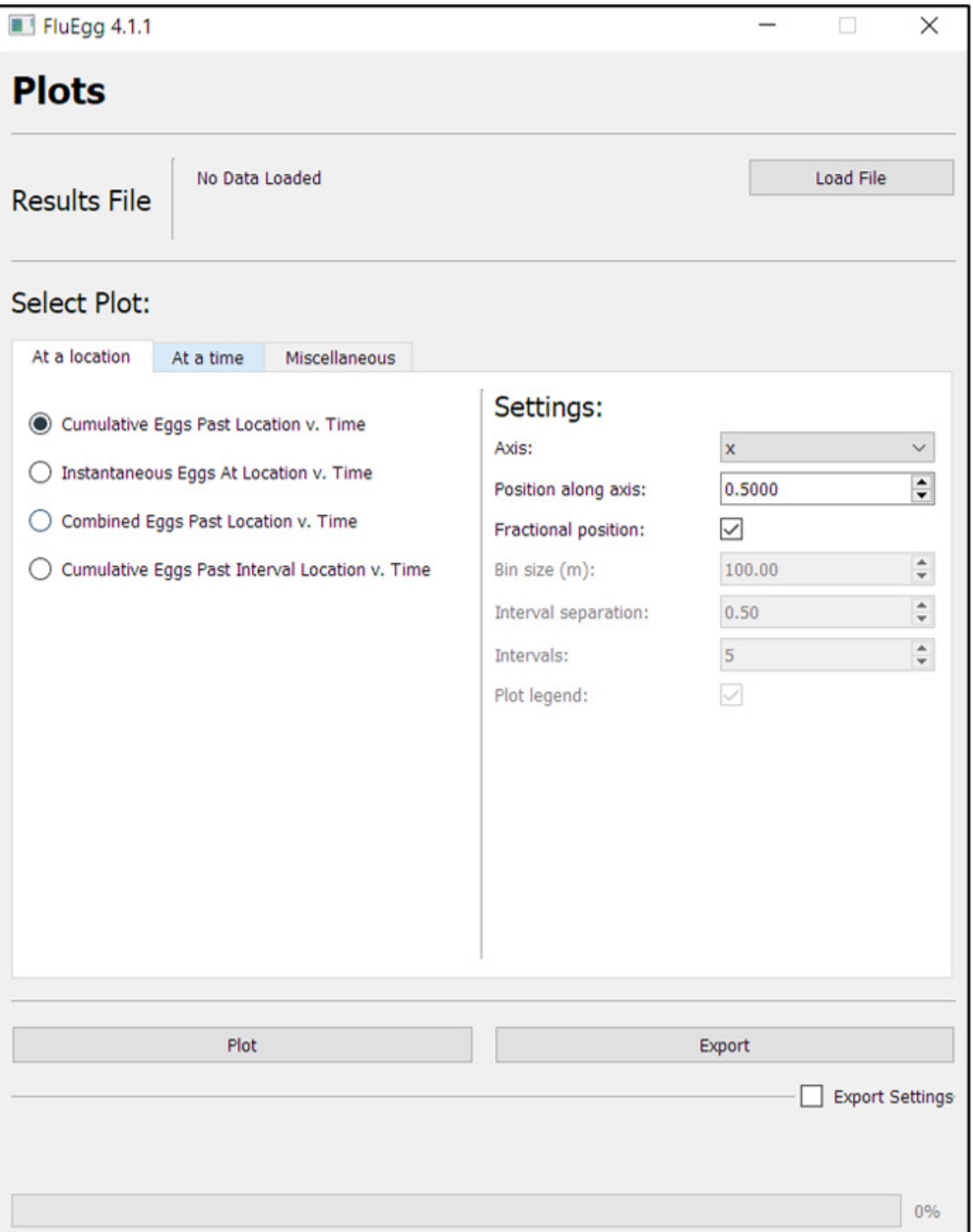

Figure 14. The "Plots" window within the Fluvial Egg Drift Simulator (FluEgg). The top panel shows the "At a location" tab, the middle panel shows the "At a time" tab, and the bottom panel shows the "Miscellaneous" tab. 
and monitoring the egg/larval plume as it passes by. In the "Settings," the "Axis" options refer to the streamwise (x), lateral (y), and vertical (z) axes of the river. The abscissae of the plots you will generate in this tab are always time. The ordinate for these plots is the fraction of the total number of eggs released expressed in decimal form. To convert to number of eggs, multiply by the total number of eggs released. For simplicity, the plot titles and axis labels refer to all simulated particles as "eggs" regardless of the developmental stage (whether they are eggs or larvae). The presets in the "At a location" tab are as follows:

1. Cumulative Eggs Past Location v. Time: A plot of the cumulative fraction of eggs that have passed a position along the $\mathrm{x}$ - (streamwise), $\mathrm{y}$ - (lateral), or z-axis (vertical) versus time.

2. Instantaneous Eggs Past Location v. Time: The fractional number of eggs at a streamwise (x-direction) location within a bin of prescribed size. This plot gives you an idea of the egg flux at a location along the river over time.

3. Combined Eggs Past Location v. Time: A superimposed plot of the cumulative fraction of eggs that have passed a streamwise (x-axis) location over time (fig. 15).

4. Cumulative Eggs Past Interval Location v. Time: Multiple superimposed "Cumulative Eggs Past Location v. Time" plots, separated along the axis by a set interval.

\section{"At a time" Plots}

The "At a time" tab provides options to plot and export information about where eggs/larvae are located within the model domain at a particular time (middle panel of fig. 14). These plots are useful if you want to look at the results at a snapshot in time (for the whole egg/larval plume). In the Settings, the "Axis" options refer to the streamwise (x), lateral (y), and vertical (z) axes of the river. The abscissa of the plots you will generate in this tab are a distance or fractional position along either the streamwise, lateral, or vertical directions. Only the $\mathrm{x}$-axis can be plotted as a distance (the y-axis and z-axis options are automatically plotted as fractional position). Note that if you select the z-axis, the abscissa and ordinate of the resulting two-dimensional plot will be transposed.

Because the last cell of the FluEgg model domain is extended infinitely to accommodate eggs that leave the hydraulic model domain, this feature will exclude eggs that leave the hydraulic model domain from analysis. If the user has loaded a full-size results file, then there is an option to exclude any eggs that have exited the hydraulic domain from the "At a time" plots to circumvent this problem. First, the user can click the "Calculate" button, located below the time presets, to identify the fraction of eggs outside of the hydraulic domain at the selected time. Then, if desired, the user can check the box for "Plot only in hydraulic domain" to exclude the eggs outside of the hydraulic model domain.
The presets in the "At a time" tab are as follows:

1. Distribution of Eggs at a Time: A plot of the distribution of the eggs/larvae at a given time. Examples of this plot for the $\mathrm{x}$-axis and $\mathrm{z}$-axis are shown in figure 16 .

a. Axis: The distribution may be over $\mathrm{x}-, \mathrm{y}-$, or z-axis.

b. Plot time (h): The time, in hours, for which the plot is generated. Can be set to hatching or gas bladder inflation using the time preset buttons.

c. Bins: The distribution is plotted as a histogram. This sets the number of bins used to create the histogram.

d. Fraction: The check for fractional (normalized) position, uncheck for real distance (real distance only allowed for the streamwise or $\mathrm{x}$-axis of the river).

2. KML Quantiles Downstream at a Time: This generates a KML file that plots the location of the eggs along the river in Google Earth ${ }^{\mathrm{TM}}$ at the specified time. Rather than creating a plot, this step exports the KML file that can be opened and viewed in Google Earth ${ }^{\mathrm{TM}}$.

a. To run this function, a KML file representing the centerline of the river must be supplied. To load the centerline file, click the "Export Settings" checkbox and then click the "Select" button next to "KML Project Path."

\section{Miscellaneous Plots}

The "Miscellaneous" tab has plotting options that do not fit as neatly into the "At a location" or "At a time" categories (bottom panel of fig. 14). The presets in the "Miscellaneous" tab are as follows:

1. Three Axis Graph: A set of three plots that show the centroid (median) plume location versus time along the $\mathrm{x}$-axis (streamwise), $\mathrm{y}$-axis (lateral), and $\mathrm{z}$-axis (vertical).

2. Location Quantiles Over Time: A plot of egg location quantiles along an axis versus time. The plotted quantiles are the minimum, 0.10 quantile, 0.25 quantile, median (0.50 quantile), 0.75 quantile, 0.90 quantile, and maximum. This plot is useful for visualizing how the shape and speed of the plume change as it drifts downstream. An example is shown in figure 17.

3. KML Centroid Downstream: This step generates a KML file that plots a point location for the downstream egg centroid for every timestep along a stream. The points are also colored based on egg depth. Rather than creating a plot, this step exports the KML file that can be opened and viewed in Google Earth ${ }^{\mathrm{TM}}$.

a. To run this function, a KML file of the centerline of the river must be supplied. To load the centerline file, click the "Export Settings" checkbox and then click the "Select" button next to "KML Project Path." 


\section{Quantity of Eggs Past 0.5 Along the X-Axis v. Time}

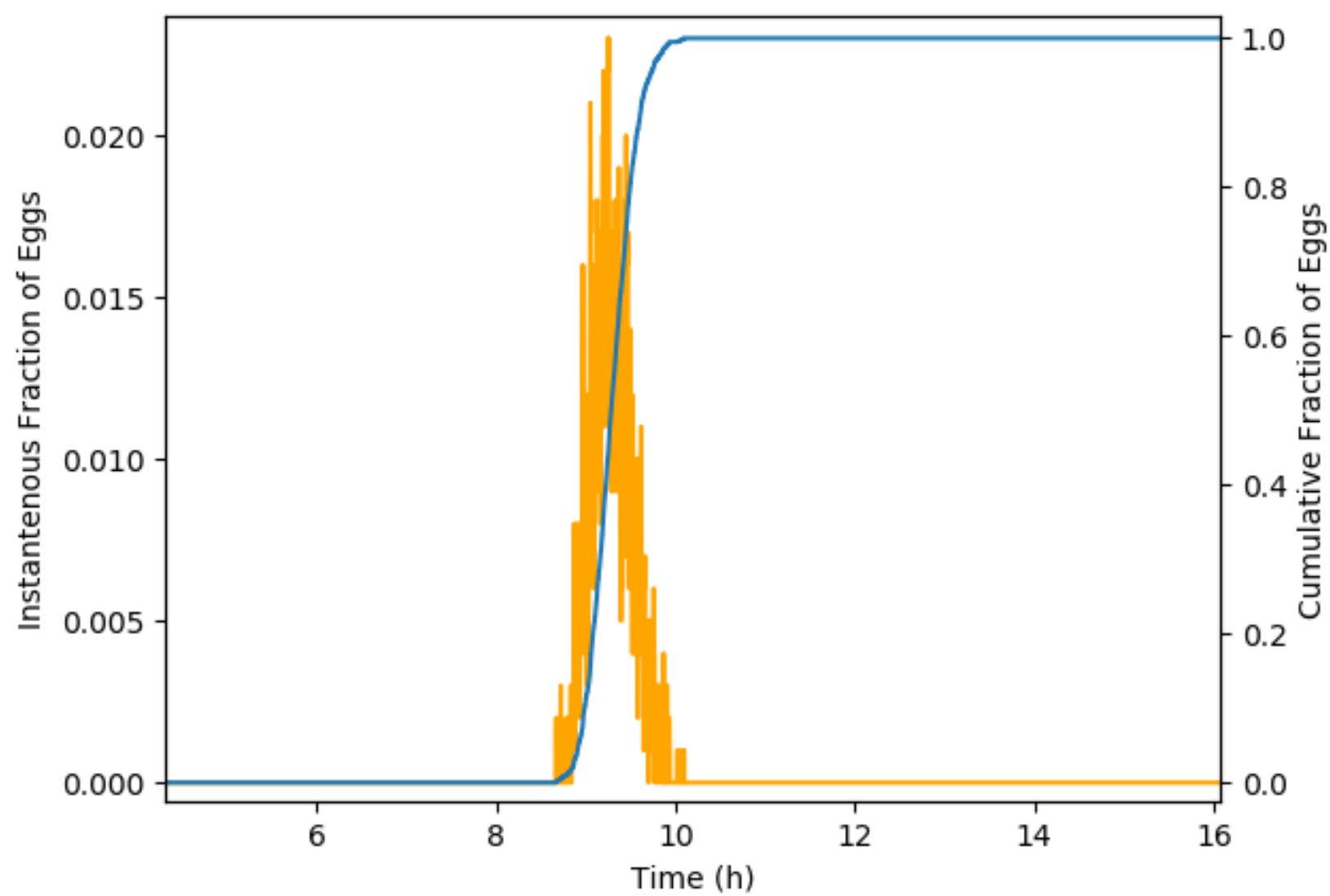

Figure 15. Example of the "Combined Eggs Past Location v. Time" plot within the Fluvial Egg Drift Simulator (FluEgg). The blue line indicates the cumulative fraction of eggs past the halfway point of the model over time. The yellow line indicates the instantaneous fraction of eggs within plus or minus 50 meters of the halfway point of the model.
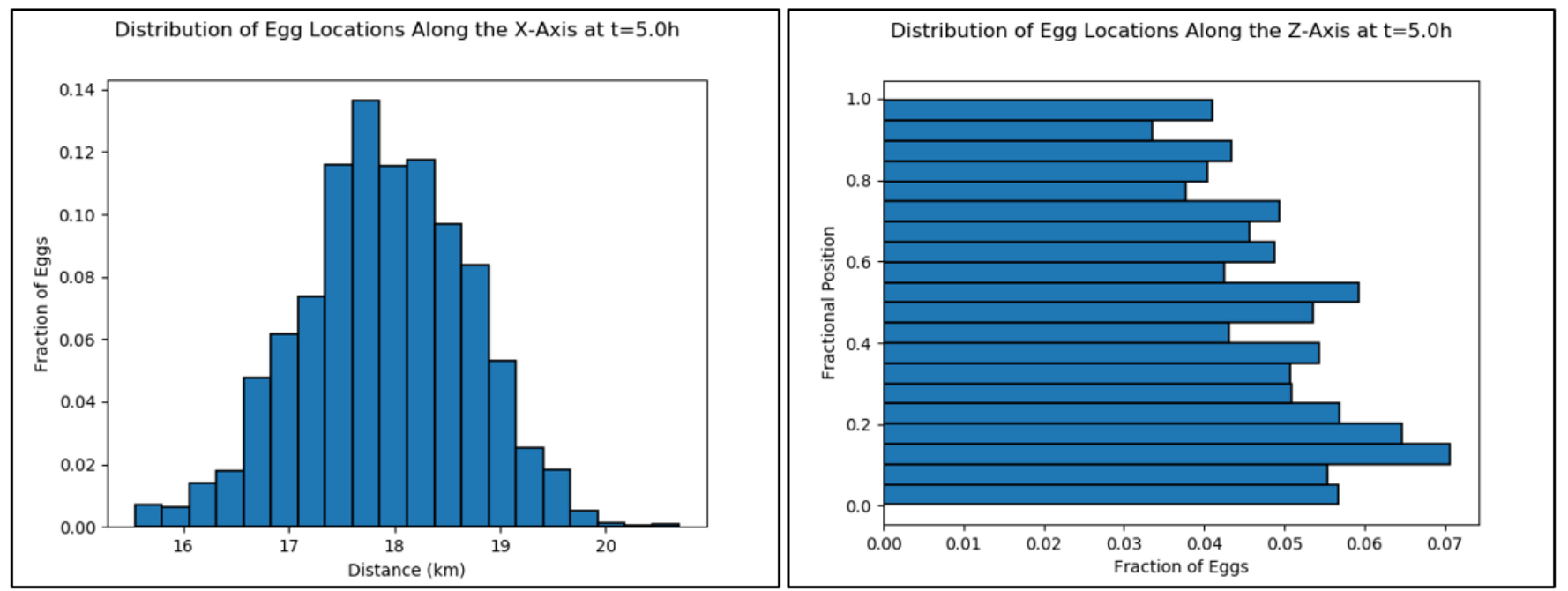

Figure 16. Example of the "Distribution of Eggs" plots within the Fluvial Egg Drift Simulator (FluEgg). The left panel shows the distribution along the $\mathrm{X}$-axis and the right panel shows the distribution along the $\mathrm{Z}$-axis at 5 hours $(\mathrm{h})$ after spawning. 


\section{Location Quantiles Along the X-Axis v. Time}

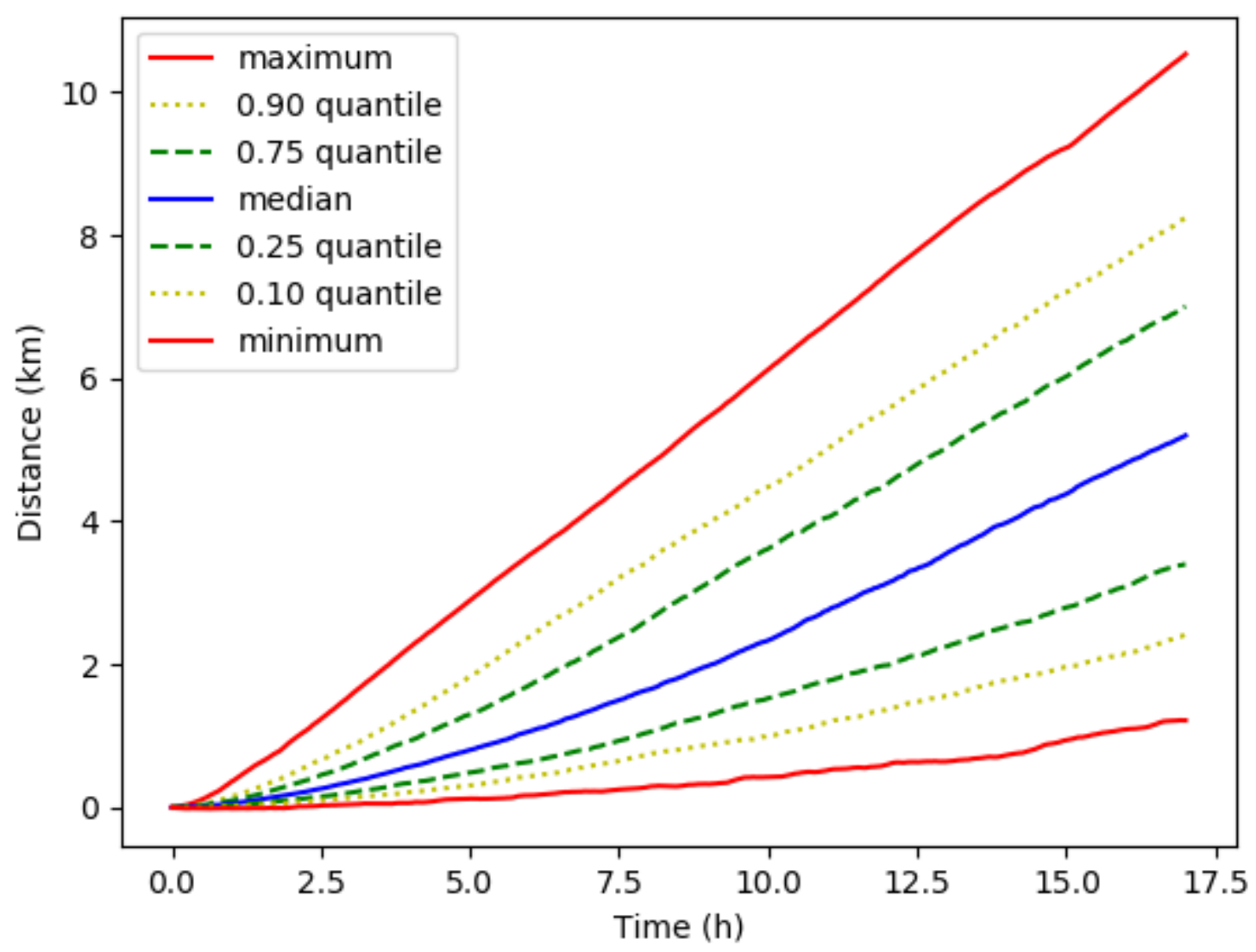

Figure 17. Example of the "Location Quantiles Over Time" plot for the X-axis within the Fluvial Egg Drift Simulator (FluEgg).

To display a plot, select the "Plot" radio button, then adjust the settings to the right, then click the "Plot" button. A new window will open, displaying an interactive plot. Multiple plot windows may be open at once. The data for any plots may be exported to CSV by clicking the "Export" button next to the "Plot" button. By default, these exports will be saved in the results directory where the simulation results are saved. To change this location, click the "Select Folder" button next to "Export Path" in the "Export Settings" section. The exported file will automatically be named based on the simulation name, the plot or export run, and metadata. To adjust the export filename, select or deselect the options next to "File Name" in the "Export Settings."

\section{Example Applications}

Four example applications of FluEgg are described in this section. The files needed to follow along with these examples can be downloaded from the FluEgg release website (examples_files.zip, https://code.usgs.gov/FluEgg/fluegg/-/releases; Domanski and Berutti, 2020).

\section{Running FluEgg with a CSV River Input File}

In this example you will load a CSV river input file into FluEgg, enter the egg and simulation parameters, and run FluEgg. First, download and save the file named "simpleriverinput.csv" or create a CSV file that matches the data in table 1 and save it to your computer. Activate FluEgg by double clicking on the executable file. In the first panel ("Hydraulic Channel"), select the radio button for "CSV," and click the "Browse" button. A window will pop up where you can navigate to where you saved the CSV river input file (either the downloaded file or the one you created) and open it. The path and filename of the river input file will populate in the box below "CSV." Select the radio button for "Parabolic-Constant" eddy diffusivity (the default). The hydraulic data needed for the FluEgg simulation are now ready.

The second panel of the FluEgg home window is for entering the egg parameters. For the initial position of the eggs, enter $\mathrm{X}=0, \mathrm{Y}=0.5$, and $\mathrm{Z}=1$ and ensure the checkboxes labeled "Fractional" to the right of the $\mathrm{Y}$ and $\mathrm{Z}$ fields are checked. Enter 5000 for the number of eggs, select "Silver" for the species, and select "Varying $\rho, \mathrm{d}$ " for the density and diameter model. 
Table 1. Data for example of comma-separated values (CSV) river input file.

[CellNumber, cell number, unitless; CumlDistance_km, cumulative distance downstream, from the most upstream point in the study reach to the downstream end of the cell, in kilometers; Depth_m, average water depth in cell, in meters; Q_cms, average water discharge in cell, in cubic meters per second; Vmag_mps, average longitudinal velocity in cell, in meters per second; Vvert_mps, average vertical velocity in cell, in meters per second; Vlat_mps, average lateral velocity in cell, in meters per second; Ustar_mps, average shear velocity, in meters per second; Temp_C, average water temperature in cell, in degrees Celsius]

\begin{tabular}{lccccllll}
\hline CellNumber & CumlDistance_km & Depth_m & O_cms & Vmag_mps & Vvert_mps & Vlat_mps & Ustar_mps & Temp_C \\
\hline 1 & 20 & 5 & 2832 & 1.72 & 0 & 0 & 0.215 & 22 \\
2 & 40 & 5 & 2832 & 1.67 & 0 & 0 & 0.20875 & 22 \\
3 & 60 & 5 & 2832 & 1.57 & 0 & 0 & 0.19625 & 22 \\
4 & 80 & 5 & 2832 & 1.89 & 0 & 0 & 0.23625 & 22 \\
5 & 100 & 5 & 2832 & 1.62 & 0 & 0 & 0.2025 & 22 \\
6 & 120 & 5 & 2832 & 1.42 & 0 & 0 & 0.1775 & 22 \\
7 & 140 & 5 & 2832 & 1.51 & 0 & 0 & 0.18875 & 22 \\
8 & 160 & 6 & 2832 & 1.18 & 0 & 0 & 0.1475 & 22 \\
9 & 180 & 7 & 2832 & 0.24 & 0 & 0 & 0.03 & 22 \\
10 & 200 & 7 & 2832 & 0.19 & 0 & 0 & 0.02375 & 22 \\
11 & 220 & 7 & 2832 & 0.16 & 0 & 0 & 0.02 & 22 \\
12 & 240 & 7 & 2832 & 0.17 & 0 & 0 & 0.02125 & 22 \\
13 & 260 & 8 & 2832 & 0.16 & 0 & 0 & 0.02 & 22 \\
14 & 280 & 8 & 2832 & 0.15 & 0 & 0 & 0.01875 & 22 \\
15 & 300 & 10 & 2832 & 0.1 & 0 & 0 & 0.0125 & 22 \\
\hline
\end{tabular}

The third panel of the FluEgg home window is for entering the simulation parameters. Select the radio button for a "Forward" simulation. Click the "Egg Hatching" duration preset to run the simulation until the time at which the eggs hatch. The duration field will autopopulate with a value of about 32.9 hours. Note that this time depends on the temperature provided in the river input file. Set the time step $(\Delta t)$ to 10 . Enter "csv-example" for the simulation name and click "Select Output Folder." In the window that opens, navigate to where you want to store the results and click "Select folder." Choose "Condensed" for the results export, then click the "Run" button.

While the simulation is running, a bar at the bottom of the simulation panel will show the progress of the simulation. When the simulation is complete, a green "csv-example saved" message will show. You can also check for the csv-example.h5 file in the folder you selected for the results. You can now click the "Open Plot Window" button and the results will be automatically loaded into the plotting window. In the "At a time" tab, select "Distribution of Eggs at a Time," set plot time to 30 hours, the number of bins to 40 , and click the "Plot" button. The resulting longitudinal (streamwise) distribution plot of the silver carp eggs should look like figure 18, though it may not look identical owing to the random-walk component of the model.

\section{Running FluEgg with a Steady HEC-RAS Project}

This example is based on a set of flume experiments designed to characterize the evolution of drifting grass carp eggs in a flume (Tang and others, 1989). These experiments were used to validate the FluEgg model upon its first release by Garcia and others (2013). These tests were run using smooth boundary conditions, which were applicable to the laboratory setting of Tang and others (1989) but are not suitable for simulating natural bodies of water. The smooth boundary simulation has since been removed from FluEgg, so the Tang and others (1989) results will not be replicated exactly in the current version. Refer to Garcia and others (2013) for the results of the validation tests of FluEgg using the experimental data of Tang and others (1989). This section simulates drifting eggs in the same flume configuration, but with rough boundary conditions, as a simple example to compare unsteady and steady HEC-RAS examples.

The first example is a simple demonstration of how to run a steady state simulation using a HEC-RAS project. The HEC-RAS model represents a rectangular laboratory flume with a Manning's $n$ of 0.0098 , a discharge of 0.1728 cubic meter per second, and a friction slope of $1.15 \mathrm{E}-04$. Activate FluEgg by double clicking on the executable file. In the "Hydraulic Channel" panel of the FluEgg window, with the Vertical Eddy Diffusivity Function set to "Parabolic-Constant," select the "HEC-RAS" radio button, then click the "Browse" button. Navigate to HEC-RASFlumeCase.prj and open it. A new window will pop up for entering the settings for using the HEC-RAS project; enter these settings to match figure 19. Note that the start time does not matter for a steady simulation. Click "Ok" to return to the FluEgg home window. Upon returning to the FluEgg home window, the "Eggs" and "Simulation" panels will become editable. Fill these panels out to match figure 20, using the hatching time preset for the 


\section{Distribution of Egg Locations Along the X-Axis at $\mathrm{t}=30.0 \mathrm{~h}$}

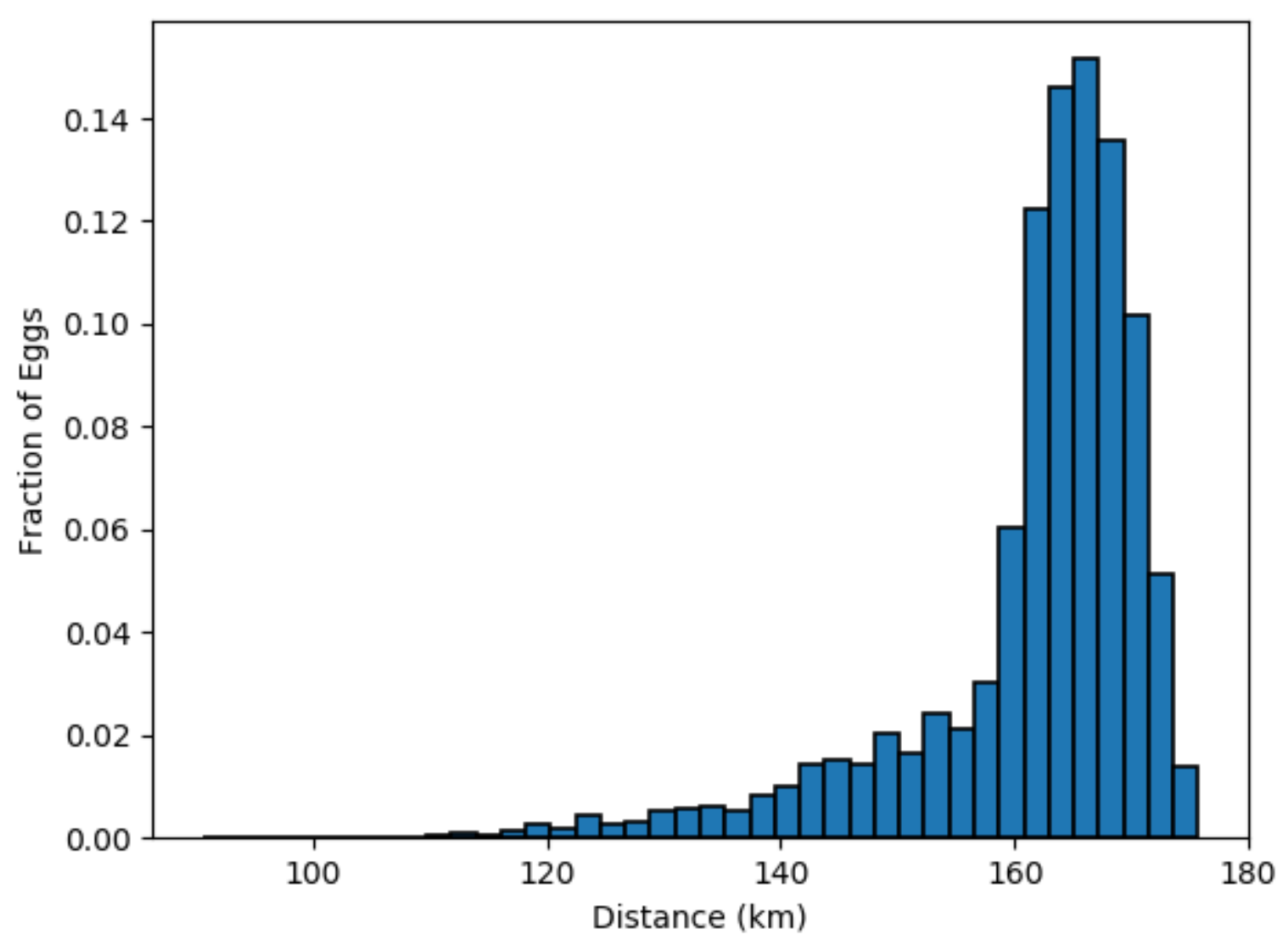

Figure 18. Distribution of eggs in the streamwise $(X)$ direction at 30 hours (close to hatching time) within the Fluvial Egg Drift Simulator (FluEgg).

simulation duration by clicking the "Egg Hatching" button. Use the "Select Output Folder" button to choose the folder for storing the results (otherwise the results will be saved to a "results" folder in the same directory as the executable file). Click the "Run" button to start the simulation. It will take several minutes to run.

When the simulation is complete, click the "Open Plot Window" button and the results will be automatically loaded into the plotting window. Choose the "At a time" tab and select the radio button for "Distribution of Eggs at a Time." Select the " $x$ " axis, set the plot time to 40.5 hours, leave the default bin size, uncheck the option for fractional distances, and click "Plot." The resulting longitudinal distribution of the eggs at hatching time indicates the plume centroid is located around $53.9 \mathrm{~km}$, with leading and tailing edges at 54.5 and $53.2 \mathrm{~km}$, respectively (fig. 21). The results will deviate somewhat from what is shown in figure 21 due to the stochastic component of the model, particularly for runs completed with fewer than 5,000 eggs. Refer to the "Plotting and Post-Processing Results" section for other visualization and export options.

\section{Running FluEgg with an Unsteady HEC-RAS Project}

To serve as an example of running the FluEgg program with river input data from an unsteady HEC-RAS simulation, a simple hydrograph is applied to the flume from the previous example as the upstream boundary condition (fig. 22). The peak discharge of the hydrograph is 0.5 cubic meter per second. The downstream boundary condition is set to the normal depth with a friction slope of 1.15E-04.

Activate FluEgg by double clicking on the executable file. In the "Hydraulic Channel" panel of the FluEgg window, select the "HEC-RAS" radio button, then click the "Browse" button. Navigate to HEC-RASFlumeCase.prj and open it. A new window will pop up for entering the settings for using the HEC-RAS project; enter these settings to match figure 23. Choose "Flume Unsteady Hydrograph" for the Plan, select the "Unsteady" radio button, and set the temperature to 22 degrees Celsius. The option for "Profile" will be grayed out because this is only an option for steady HEC-RAS models. Choose the spawning time as January 1, 2017, at 12:00 a.m. Click 


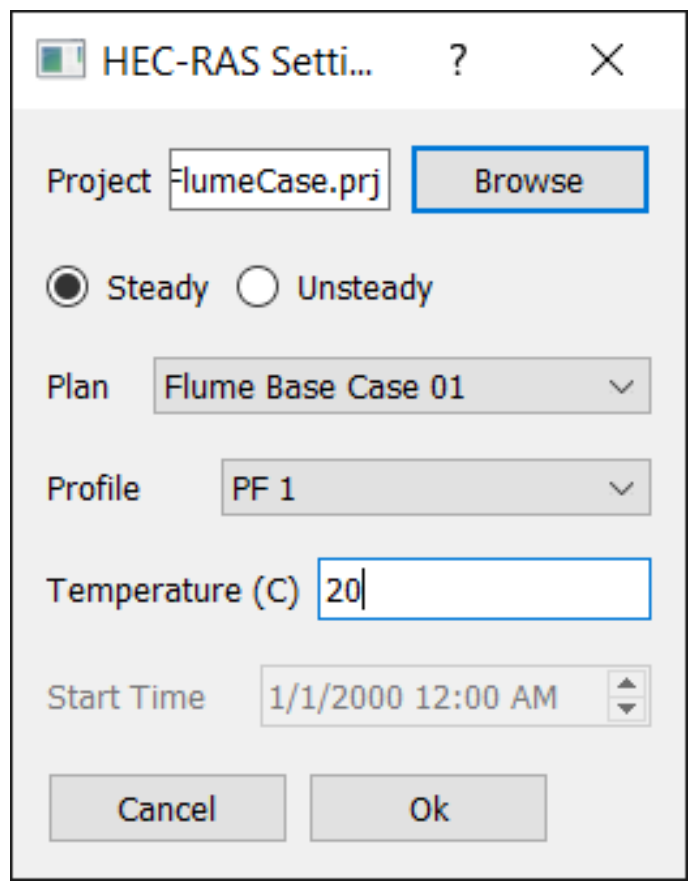

Figure 19. Hydrologic Engineering Center's River Analysis System (HEC-RAS) Settings window for the steady flume example within the Fluvial Egg Drift Simulator (FluEgg).

"Ok" to return to the FluEgg home window. Upon returning to the FluEgg home window, the "Eggs" and "Simulation" panels will become editable. Fill these panels out to match figure 24, using the hatching time preset for the simulation duration. Note that the egg hatching time (32.6 hours) for a water temperature of 22 degrees Celsius is shorter than it was in the previous example in which the water temperature was 20 degrees Celsius (40.6 hours). Use the "Select Output Folder" button to choose the folder for storing the results (otherwise the results will be saved to a "results" folder in the same directory as the executable file). Click the "Run" button to start the simulation. It will take several minutes to run.

When the simulation is complete, click the "Open Plot Window" button and the results will be automatically loaded into the plotting window. Choose the "At a time" tab and select the radio button for "Distribution of Eggs at a Time." Select the "x" axis, set the plot time to 32.5 hours, leave the default bin size, uncheck the option for fractional distances, and click the "Plot" button. The resulting longitudinal distribution of the eggs at hatching time indicates the plume centroid is located around $30.8 \mathrm{~km}$, with leading and tailing edges at 29.3 and $31.7 \mathrm{~km}$, respectively (fig. 25). The results will deviate somewhat from what is shown in fig. 25 due to the stochastic component of the model, particularly for runs completed with fewer than 5,000 eggs. Refer to the "Plotting and Post-Processing Results" section for other visualization and export options.

\section{Generating KML Outputs in FluEgg - Illinois River Example}

In this example you will first use a CSV river input file to run a FluEgg simulation for the Illinois River, then use those results to explore the KML generating utilities in FluEgg. First, download and save the files named "IllinoisRiver_5Year_22deg. csv" (CSV river input file) and "IllinoisRiver-centerline. kml" (KML centerline file for the Illinois River). The CSV river input file was created using the output of a steady flow HEC-RAS simulation of the Illinois River for a flow with an annual exceedance probability of 20 percent (5-year recurrence interval). This flow corresponds to 63,500 cubic feet per second at the USGS streamgage 05543500 (Illinois River at Marseilles, Illinois; Soong and others, 2004). The water temperature is 22 degrees Celsius. Load "IllinoisRiver_5Year_22deg.csv" into FluEgg and fill out the egg and simulation parameters to match figure 26, selecting "Egg Hatching" in the "Duration Presets." Click the "Select Output Folder" button to choose where to store the $\mathrm{h} 5$ results file, then click the "Run" button. The simulation will take several minutes to run.

When the simulation is complete, click the "Open Plot Window" button at the bottom of the FluEgg home window. If you ran the simulation previously, then open FluEgg, click the "Open Plot Window" button, and load the h5 results file by clicking the "Load File" at the top right. Select the "At a time tab" and click the radio button for the "KML Quantiles Downstream at a Time (Export only)" option. Click the "Egg Hatching" time preset to set the plot time to the timestep closest to the hatching time.

Click the "Export Settings" checkbox and additional options will become visible at the bottom of the window. Click the "Select Folder" button to choose where the exported KML file will be stored and click the checkboxes for the desired setting for the filename. Next, load the river centerline file (IllinoisRiver-centerline.kml) by clicking the "Select" button for the KML Project Path (located at the bottom right) and navigating to where that file was stored. The plotting window should look similar to figure 27.

Click the "Export" button. A KML file will be exported that marks the spawning location and the locations of the following quantiles: 0 (tailing edge), 0.1, 0.25, 0.5 (median), 0.75, 0.9, and 1.0 (leading edge). The filename will vary depending on the filename options selected but will include the phrase "downstream position quantiles." Click "Save" in the dialog box that opens. Navigate to the file and double click to open it in Google Earth.

The second KML plotting utility is the "KML Centroid Downstream (Export Only)" option located in the "Miscellaneous" tab of the plotting window. Select this option and click the "Export" button (the centerline file was already loaded in the previous step). A KML file will be exported that shows the centroid position at each time step of the simulation. These data are represented as a series of points along the streamline, with each point representing the location of the centroid at one of the time steps. The filename will vary depending on the filename options selected but will include the phrase "centroid position downstream and fractional depth." Click "Save" in the dialog box that opens. Navigate to the file and double click to open it in Google Earth. 
2) Eggs

Initial Position: $X(\mathrm{~km}) 0$

Y 0.5

Fractional

\begin{tabular}{l|l} 
Z & 1
\end{tabular}

Fractional

Number of Eggs: 1000

Species: $\bigcirc$ grass $\bigcirc$ Silver $\bigcirc$ Bighead

Density and Diameter Model: $($ Varying $\rho, d \bigcirc$ Constant $\rho, d$

Density $\left(\mathrm{kg} / \mathrm{m}^{3}\right):$ Diameter $(\mathrm{mm})$ :

3) Simulation

Simulation Direction: $\bigcirc$ Forward $\bigcirc$ Reverse

Duration Presets: Egg Hatching Gas Bladder Inflation

Duration (h): 40.55512903076613

$\Delta \mathrm{t}(\mathrm{s}): 0.5$

Simulation Name: steadyflume Select Output Folder

Results Export: $\bigcirc$ Full Size $\bigcirc$ condensed

Run

Figure 20. Egg and simulation parameters for the steady flume example within the Fluvial Egg Drift Simulator (FluEgg). 
Distribution of Egg Locations Along the X-Axis at $t=40.5 \mathrm{~h}$

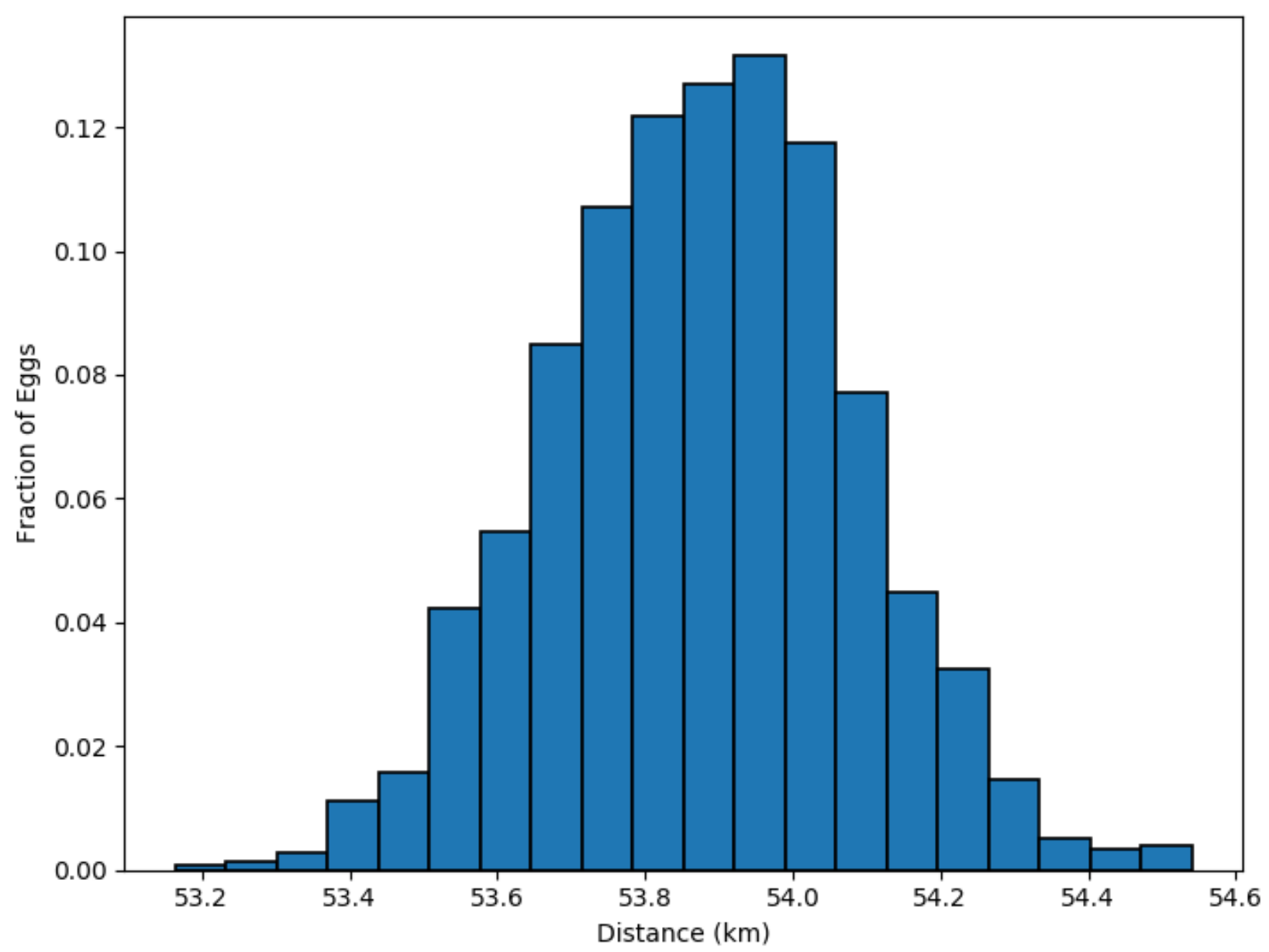

Figure 21. The resulting distribution of grass carp eggs at the hatching time in the steady flume example with a water temperature of 20 degrees Celsius within the Fluvial Egg Drift Simulator (FluEgg). 


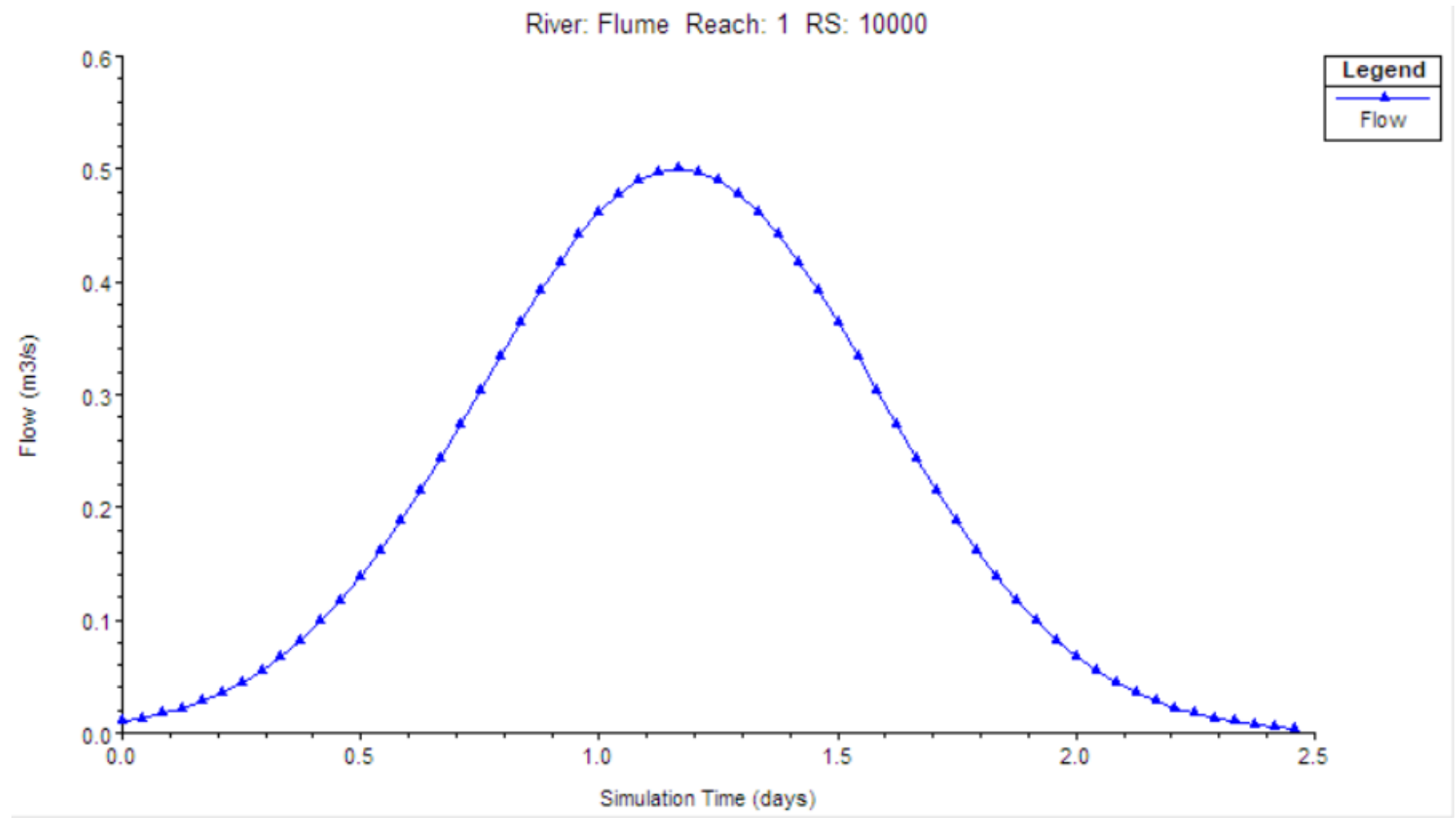

Figure 22. The simple hydrograph used for the unsteady flume example within the Fluvial Egg Drift Simulator (FluEgg). The start time of the simulation is January 1, 2017, at 12:00 a.m.

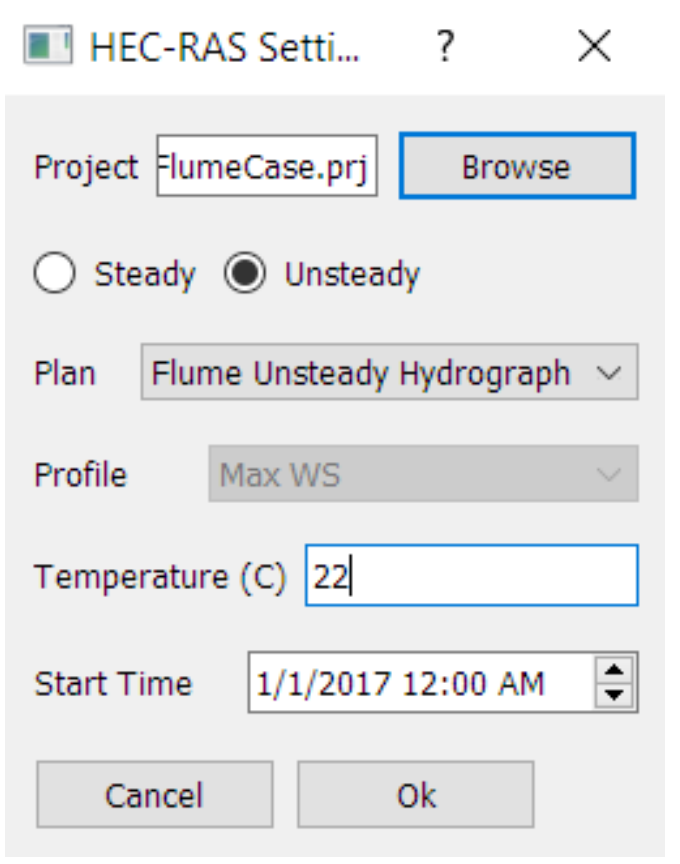

Figure 23. Hydrologic Engineering Center's River Analysis System (HEC-RAS) Settings for the unsteady flume example within the Fluvial Egg Drift Simulator (FluEgg). 


\section{2) Eggs}

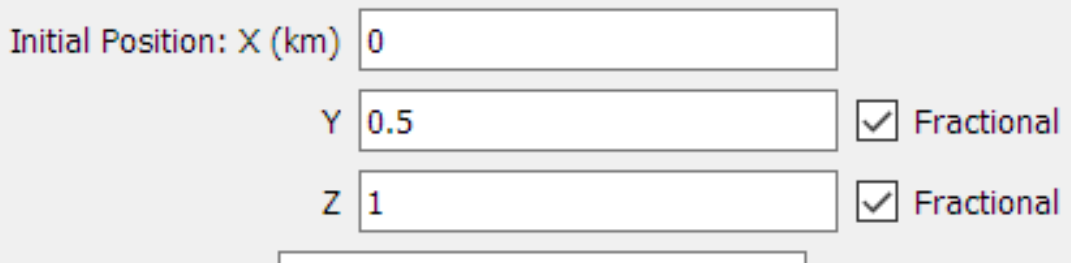

Number of Eggs: 1000

Species: $\bigcirc$ Grass $\bigcirc$ Silver $\bigcirc$ Bighead

Density and Diameter Model: $(2$ Varying $p, d \bigcirc$ Constant $p, d$

Density $\left(\mathrm{kg} / \mathrm{m}^{3}\right)$ :

Diameter ( $\mathrm{mm})$ :

3) Simulation

Simulation Direction: $\bigcirc$ Forward $\bigcirc$ Reverse

Duration Presets: Egg Hatching Gas Bladder Inflation

Duration (h): 32.60959192536191

$\Delta \mathrm{t}(\mathrm{s}): 0.25$

Simulation Name: unsteadyflume Select Output Folder

Results Export: $\bigcirc$ Full Size $\bigcirc$ Condensed

Run

Figure 24. Egg and simulation parameters for the unsteady flume example within the Fluvial Egg Drift Simulator (FluEgg). 
Distribution of Egg Locations Along the X-Axis at $t=32.5 \mathrm{~h}$

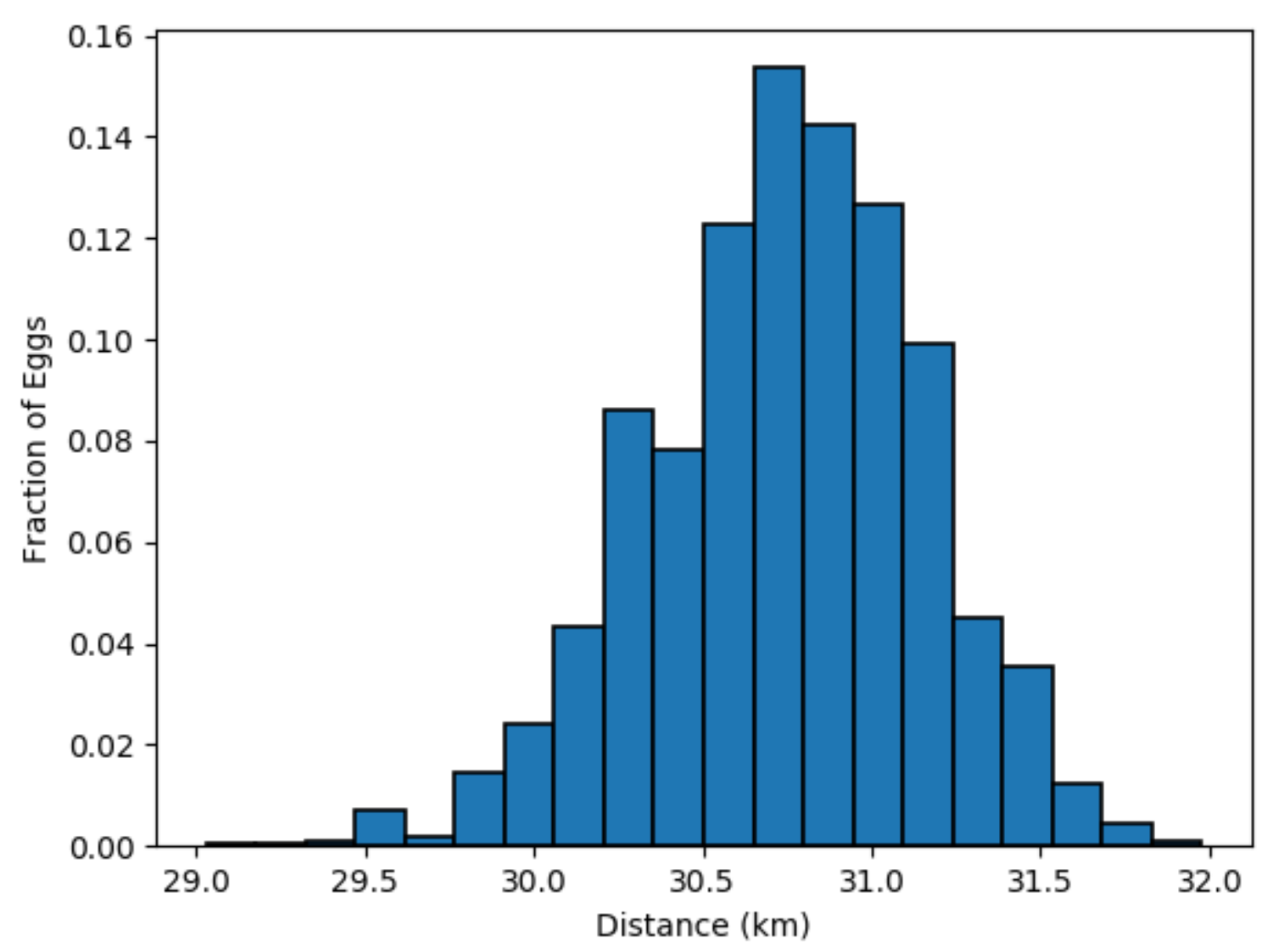

Figure 25. The resulting longitudinal distribution of grass carp eggs at the hatching time in the unsteady flume example with a water temperature of 22 degrees Celsius within the Fluvial Egg Drift Simulator (FluEgg). 


\section{Egg Simulation}

Batch Simulation

1) Hydraulic Channel

(2) csv

leroy/Downloads/IllinoisRiver_5Year_22deg.csv hecras project

Browse

Vertical Eddy Diffusivity Function: $($ Parabolic-Constant $\bigcirc$ Parabolic $\bigcirc$ constant

2) Eggs

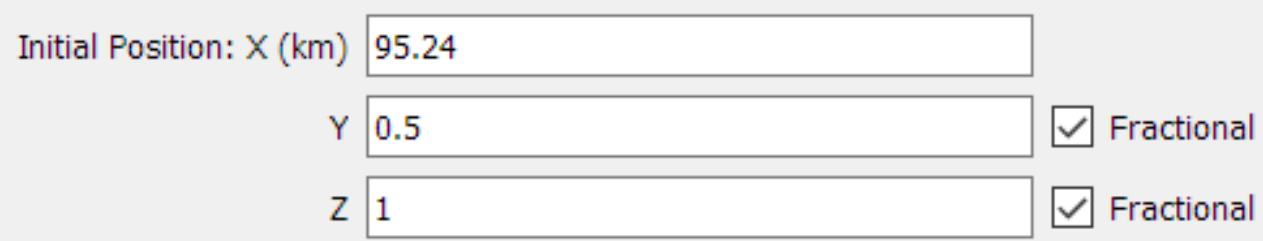

Number of Eggs: 5000

Species: $\bigcirc$ Grass $\bigcirc$ Silver $\bigcirc$ Bighead

Density and Diameter Model: $($ Varying $\rho, d \bigcirc$ Constant $\rho, d$

Density $\left(\mathrm{kg} / \mathrm{m}^{3}\right)$ :

Diameter ( $\mathrm{mm})$ :

3) Simulation

Simulation Direction: $\bigcirc$ Forward $\bigcirc$ Reverse

Duration Presets: Egg Hatching Gas Bladder Inflation

Duration (h): 32.88877663392344

$\Delta \mathrm{t}(\mathrm{s}): 5$

Simulation Name: illinoisriver_5yr_22deg $\quad$ Select Output Folder

Results Export: $\bigcirc$ Full Size $\bigcirc$ condensed

Run

Channel Loaded

Figure 26. FluEgg home window with egg and simulation parameters for Illinois River example within the Fluvial Egg Drift Simulator (FluEgg). 


\section{Plots}

Results File

C:/.../Desktop/results/illinoisriver_5yr_22deg.h5

Load File

5000 silver eggs over 118400 s, with a timestep of 5 s (23680 steps).

\section{Select Plot:}

\begin{tabular}{|c|c|c|c|c|c|}
\hline At a location & At a time & Miscellaneous & & & \\
\hline Distributio & fFone at = & & Settings: & & \\
\hline & & & Axis: & $\mathrm{x}$ & $\checkmark$ \\
\hline () KML Quar & s Downstre & at a Time (Export only) & Plot time (h): & 30.000 & $\div$ \\
\hline & & & & & \\
\hline & & & Time presets: & $\mathrm{Ga}$ & \\
\hline & & & Fraction of eggs out of domain: & & \\
\hline & & & Plot only in hydraulic domain: & $\square$ & \\
\hline & & & Bins: & 20 & $\div$ \\
\hline & & & Fractional: & $\square$ & \\
\hline
\end{tabular}

Plot

Export

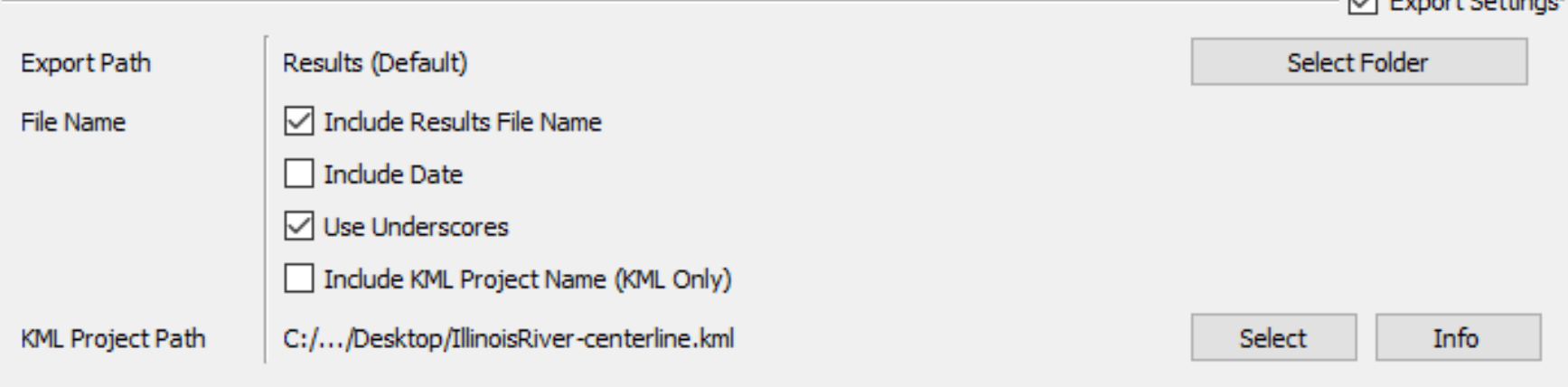

Figure 27. Plotting window for exporting a Keyhole Markup Language (KML) file of the plume quantiles at 30 hours after spawning for the Illinois River example within the Fluvial Egg Drift Simulator (FluEgg). 


\section{References Cited}

Babaeyan-Koopaei, K., Ervine, D.A., Carling, P.A., and Cao, Z., 2002, Velocity and turbulence measurements for two overbank flow events in River Severn: Journal of Hydraulic Engineering, v. 128, no. 10, p. 891-900, accessed July 15, 2020, at https://doi.org/10.1061/(ASCE)07339429(2002)128:10(891).

Biron, P.M., Robson, C., Lapointe, M.F., and Gaskin, S.J., 2004, Comparing different methods of bed shear stress estimates in simple and complex flow fields: Earth Surface Processes and Landforms, v. 29, no. 11, p. 1403-1415, accessed July 15, 2020, at https://doi.org/10.1002/esp.1111.

Chapman, D.C., and George, A.E., 2011, Developmental rate and behavior of early life stages of bighead carp and silver carp: U.S. Geological Survey Scientific Investigations Report 2011-5076, 11 p., accessed December 7, 2017, at https://doi.org/10.3133/sir20115076.

Domanski, M.M., and Berutti, M.C., 2020, FluEgg: U.S. Geological Survey software release, https://doi.org/10.5066/ P93UCQR2.

Embke, H.S., Kocovsky, P.M., Garcia, T., Mayer, C.M., and Qian, S.S., 2019, Modeling framework to estimate spawning and hatching locations of pelagically spawned eggs: Canadian Journal of Fisheries and Aquatic Sciences, v. 76, no. 4, p. 597-607, accessed July 15, 2020, at https://doi.org/ 10.1139/cjfas-2018-0047.

Garcia, T., Jackson, P.R., Murphy, E.A., Valocchi, A.J., and Garcia, M.H., 2013, Development of a Fluvial Egg Drift Simulator to evaluate the transport and dispersion of Asian carp eggs in rivers: Ecological Modelling, v. 263, no. 10, p. 211-222, accessed December 7, 2017, at https://doi.org/ 10.1016/j.ecolmodel.2013.05.005.

Garcia, T., Murphy, E.A., Jackson, P.R., and Garcia, M.H., 2015, Application of the FluEgg model to predict transport of Asian carp eggs in the Saint Joseph River (Great Lakes tributary): Journal of Great Lakes Research, v. 41, no. 2, p. 374-386, accessed December 7, 2017, at https://doi.org/ 10.1016/j.jglr.2015.02.003.

George, A.E., and Chapman, D.C., 2013, Aspects of embryonic and larval development in bighead carp Hypophthalmichthys nobilis and silver carp Hypophthalmichthys molitrix: PLoS One, v. 8, no. 8, e73829. [Also available at https://doi.org/10.1371/ journal.pone.0073829.]
George, A.E., and Chapman, D.C., 2015, Embryonic and larval development and early behavior in grass carp, Ctenopharyngodon idella-Implications for recruitment in rivers: PLoS One, v. 10, no. 3, e0119023. [Also available at https://doi.org/10.1371/journal.pone.0119023.]

George, A.E., Garcia, T., and Chapman, D.C., 2017, Comparison of size, terminal fall velocity, and density of bighead carp, silver carp, and grass carp eggs for use in drift modeling: Transactions of the American Fisheries Society, v. 146, no. 5, p. 834-843. [Also available at https://doi.org/ 10.1080/00028487.2017.1310136.]

Keulegan, G.H., 1938, Laws of turbulent flow in open channels: U.S. Department of Commerce, Journal of Research of the National Bureau of Standards, v. 21, p. 707-741. [Also available at https://nvlpubs.nist.gov/nistpubs/jres/21/ jresv21n6p707_A1b.pdf.]

Murphy, E.A., Garcia, T., Jackson, P.R., and Duncker J.J., 2016, Simulation of hypothetical Asian carp egg and larvae development and transport in the Lockport, Brandon Road, Dresden Island, and Marseilles Pools of the Illinois Waterway by use of the fluvial egg drift simulator (FluEgg) model: U.S. Geological Survey Open-File Report 2016-1011, 19 p., accessed December 7, 2017, at https://doi.org/10.3133/ofr20161011.

Murphy, E.A., and Jackson, P.R., 2013, Hydraulic and waterquality data collection for the investigation of Great Lakes tributaries for Asian carp spawning and egg-transport suitability: U.S. Geological Survey Scientific Investigations Report 2013-5106, 30 p., accessed December 7, 2017, at https://doi.org/10.3133/sir20135106.

Prada, A.F., George, A.E., Stahlschmidt, B.H., Chapman, D.C., and Tinoco, R.O., 2018, Survival and drifting patterns of grass carp eggs and larvae in response to interactions with flow and sediment in a laboratory flume: PLoS One, v. 13, no. 12, e0208326. [Also available at https://doi.org/10.1371/ journal.pone.0208326.]

Rutherford, J.C., 1994, River mixing: Chichester, U.K., John Wiley \& Sons, 348 p.

Seo, I.W., and Baek, K.O., 2004, Estimation of the longitudinal dispersion coefficient using the velocity profile in natural streams: Journal of Hydraulic Engineering, v. 130, no. 3, p. 227-236. [Also available at https://doi.org/10.1061/ (ASCE)0733-9429(2004)130:3(227).]

Soong, D.T., Ishii, A.L., Sharpe, J.B., and Avery, C.F., 2004, Estimating flood-peak discharge magnitudes and frequencies for rural streams in Illinois: U.S. Geological Survey Scientific Investigations Report 2004-5103, 147 p. [Also available at https://doi.org/10.3133/sir20045103.] 
Tang, M., Huang, D., Huang, L., Xiang, F., and Yin, W., 1989, Preliminary forecast of hydraulic characteristic test of grass, green, silver carp, bighead carp egg incubation conditions in the Three Gorges Reservoir Area: Reservoir Fisheries, v. 4, p. 26-30. [In Chinese.]
Zhu, Z., Soong, D.T., Garcia, T., Behrouz, M.S., Butler, S.E., Murphy, E.A., Diana, M.J., Duncker, J.J., and Wahl, D.H., 2018, Using reverse-time egg transport analysis for predicting Asian carp spawning grounds in the Illinois River: Ecological Modelling, v. 384, p. 53-62, accessed July 15, 2020, at https://doi.org/10.1016/j.ecolmodel.2018.06.003. 
For more information about this publication, contact

Director, USGS Central Midwest Water Science Center 405 North Goodwin

Urbana, IL 61801

217-328-8747

For additional information, visit https://www.usgs.gov/centers/cm-water

Publishing support provided by the Rolla and Lafayette Publishing Service Centers 


\section{$\frac{\mathbb{3}}{3}$}

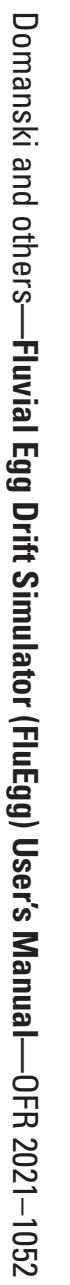

